\title{
Selective glutamine metabolism inhibition in tumor cells improves antitumor T lymphocyte activity in triple-negative breast cancer
}

\author{
Deanna N. Edwards, ${ }^{1}$ Verra M. Ngwa, ${ }^{2}$ Ariel L. Raybuck, ${ }^{3}$ Shan Wang, ${ }^{1}$ Yoonha Hwang, ${ }^{1}$ Laura C. Kim, ${ }^{2}$ Sung Hoon Cho, ${ }^{3}$ \\ Yeeun Paik, ${ }^{3}$ Qingfei Wang, ${ }^{4,5}$ Siyuan Zhang, ${ }^{4,5}$ H. Charles Manning, ${ }^{6,7,8,9,10}$ Jeffrey C. Rathmell, ${ }^{3,10,11}$ Rebecca S. Cook, ${ }^{2,10,12}$ \\ Mark R. Boothby, ${ }^{1,2,3,11}$ and Jin Chen $1,2,10,11,12,13$
}

'Division of Rheumatology and Immunology, Department of Medicine, Vanderbilt University Medical Center, Nashville, Tennessee, USA. ²Program in Cancer Biology, Vanderbilt University, Nashville, Tennessee, USA. ${ }^{3}$ Department of Pathology, Microbiology and Immunology, Vanderbilt University Medical Center, Nashville, Tennessee, USA. ${ }^{4}$ Department of Biological Sciences, College of Science, University of Notre Dame, Notre Dame, Indiana, USA. ${ }^{5}$ Mike and Josie Harper Cancer Research Institute, University of Notre Dame, South Bend, Indiana, USA. ${ }^{6}$ Department of Chemistry, ${ }^{7}$ Center for Molecular Probes, ${ }^{8}$ Vanderbilt Institute for Imaging Sciences, ${ }^{9}$ Department of Radiology and Radiological Sciences, ${ }^{10}$ Vanderbilt-Ingram Cancer Center, ${ }^{11}$ Vanderbilt Institute for Infection, Immunology and Inflammation, and ${ }^{12}$ Department of Cell and Developmental Biology, Vanderbilt University Medical Center, Nashville, Tennessee, USA. ${ }^{13}$ Veterans Affairs Medical Center, Tennessee Valley Healthcare System, Nashville, Tennessee, USA.

\begin{abstract}
Rapidly proliferating tumor and immune cells need metabolic programs that support energy and biomass production. The amino acid glutamine is consumed by effector T cells and glutamine-addicted triple-negative breast cancer (TNBC) cells, suggesting that a metabolic competition for glutamine may exist within the tumor microenvironment, potentially serving as a therapeutic intervention strategy. Here, we report that there is an inverse correlation between glutamine metabolic genes and markers of T cell-mediated cytotoxicity in human basal-like breast cancer (BLBC) patient data sets, with increased glutamine metabolism and decreased T cell cytotoxicity associated with poor survival. We found that tumor cell-specific loss of glutaminase (GLS), a key enzyme for glutamine metabolism, improved antitumor T cell activation in both a spontaneous mouse TNBC model and orthotopic grafts. The glutamine transporter inhibitor V-9302 selectively blocked glutamine uptake by TNBC cells but not CD8 ${ }^{+} \mathrm{T}$ cells, driving synthesis of glutathione, a major cellular antioxidant, to improve $\mathrm{CD8} 8^{+} \mathrm{T}$ cell effector function. We propose a "glutamine steal" scenario, in which cancer cells deprive tumor-infiltrating lymphocytes of needed glutamine, thus impairing antitumor immune responses. Therefore, tumor-selective targeting of glutamine metabolism may be a promising therapeutic strategy in TNBC.
\end{abstract}

\section{Introduction}

Rapidly dividing cells display unique metabolic traits that support their enhanced energy requirements and ever-increasing biomass (amino acids, nucleotides, lipids, etc.) synthesis to enable continued proliferation (1-9). The ability of cancer cells to hijack these metabolic processes is a known hallmark of cancer (10). Although glucose metabolism has received substantial attention, the role of amino acid metabolism in tumorigenesis and tumor progression has started to be explored more recently (11-14). Some cancer types are particularly dependent on metabolism of the amino acid glutamine for tumor cell survival and/or proliferation. For example, triple-negative breast cancers (TNBCs), which do not express estrogen receptor (ER), progesterone receptor (PR), or human epidermal growth factor (EGF) receptor 2 (HER2), are particularly "addicted" to glutamine as compared with other breast cancer subtypes $(15,16)$. TNBCs often express high levels of ASCT2, a

Conflict of interest: The authors have declared that no conflict of interest exists. Copyright: ( 2021, American Society for Clinical Investigation.

Submitted: May 8, 2020; Accepted: December 10, 2020; Published: February 15, 2021 Reference information: J Clin Invest. 2021;131(4):e140100.

https://doi.org/10.1172/JCl140100. glutamine transporter, and glutaminase (GLS), the enzyme that catalyzes the rate-limiting step in glutaminolysis, the conversion of glutamine into glutamate (17-19). Unlike other breast cancer subtypes, TNBCs do not have the benefit of molecularly targeted therapies, relying primarily on chemotherapeutic regimens. However, a growing body of evidence demonstrates that glutamine is required for TNBC tumor cell survival (17-20), suggesting that glutamine metabolism may represent a therapeutic target in TNBC.

Like tumor cells, activation of T lymphocytes increases glutamine uptake and metabolism to support mitochondrial anaplerosis, nucleotide synthesis, amino acid production, and redox balance (21). Ligation of CD3 and CD28 on T cells, an event that drives $\mathrm{T}$ cell expansion and activation, leads to upregulation of glutamine transporters such as ASCT2, SNAT1, and SNAT2 to facilitate glutamine uptake by $\mathrm{T}$ cells (22-24). Further, glutamine starvation of $\mathrm{T}$ cells significantly hinders $\mathrm{T}$ cell proliferation and cytokine production (22), illustrating the critical nature of glutamine metabolism for $\mathrm{T}$ cell-mediated immune responses. Although ASCT2 loss from $\mathrm{T}$ cells did not appear to limit $\mathrm{T}$ cell proliferation or cytotoxicity in the acute setting of viral infection in vivo (25), chronic GLS inhibition blocked conversion of glutamine to glutamate and reduced long-term CAR-T cell responses in an 
in vivo tumor model (26). Of note, immunosuppressive $\mathrm{T}$ regulatory cells (Tregs) are able to maintain reserve respiratory capacity and thrive even in the absence of glutamine (27). Together, these studies suggest that glutamine supply to and metabolism in T cells influence the nature and potency of immune responses, but also suggest that glutamine transporter blockade may not fully impede glutamine uptake into T cells.

The increased metabolic demands of tumor cells and activated $\mathrm{T}$ lymphocytes may introduce competition for glutamine within the tumor microenvironment $(8,28-30)$, creating a scenario in which tumor cells out-compete $\mathrm{T}$ cells for local glutamine and thereby alter the characteristics of the tumor-infiltrating lymphocytes (TILs). Thus, in this scenario the nutrient consumption would both promote proliferation and survival of tumor cells and simultaneously limit the capacity for T cell-mediated antitumor immunity, similar to observations with glucose $(31,32)$. Accordingly, we hypothesized that glutamine-dependent tumors, such as TNBCs, might benefit therapeutically from inhibition of glutamine metabolism, improving antitumor $\mathrm{T}$ cell responses by reversing a tumor "glutamine steal" phenomenon.

In this report, we analyzed human clinical basal-like breast cancer (BLBC) data sets and found an inverse relationship between glutamine metabolism and $\mathrm{T}$ cell cytotoxicity markers and a poor overall survival in patients whose tumors harbor both high glutamine metabolism and low $\mathrm{T}$ cell cytotoxicity signatures. Using spontaneous genetically engineered and orthotopically grafted mouse models of TNBC, we show that tumor cell-specific GLS loss increased tumor interstitial glutamine concentration and intratumoral $\mathrm{T}$ cell infiltration and activity. Pharmacological inhibition of glutamine uptake using the glutamine transporter inhibitor, V-9302, blocked glutamine uptake in tumor cells but not in T cells, which adapted through compensatory upregulation of another glutamine transporter, resulting in superior $\mathrm{T}$ cell responses within tumors while diminishing tumor growth. Together, these data indicate that tumor-selective blockade of glutamine uptake may be a feasible, molecularly targeted approach to combat TNBC, providing a 2-pronged attack that boosts antitumor immune responses while concurrently crippling tumor cell metabolism.

\section{Results}

Expression of glutamine metabolism genes inversely correlate with cytotoxic gene signatures in human BLBC. To examine if tumor cell glutamine metabolism may negatively affect antitumor immunity in TNBC, we analyzed BLBC expression data sets curated by The Cancer Genome Atlas (TCGA). BLBCs are the molecularly defined breast cancer subtype characterizing most TNBC specimens (33). Gene expression values for genes encoding glutamine-utilizing enzymes (GLS, ASNS, and GFPT1) as well as glutamine transporters (ASCT2/SLC1A5, SNAT1/SLC38A1, SNAT2/SLC38A2, and $\left.\mathrm{ATB}^{0,+} / S L C 6 A 14\right)$ were used to generate a glutamine metabolism gene signature (Figure 1A). To avoid a potentially confounding bias from more highly glycolytic tumor samples, a glycolysis signature consisting of expression values for the genes encoding glycolysis enzymes was used to identify those tumor samples with reduced glycolysis (Figure 1A). The resulting "glutamine metabolism high" subset of patient samples exhibited a significantly stronger expression profile for glutamine metabolism than glycol- ysis, while all others are classified as having "mixed metabolism." A CTL gene signature was generated using 4 genes often associated with increased $\mathrm{CD}^{+} \mathrm{T}$ cell cytotoxic activity, GZMA, GZMB, $P R F 1$, and IFNG. Correlation analyses of the glutamine metabolism high samples revealed a strong negative correlation between the glutamine metabolism and CTL gene signatures (Figure 1B, black). The same relationship between the 2 parameters was not observed in the mixed metabolism samples (Figure 1B, pink). Similarly, inverse correlations were observed between $G L S$, the rateliminting enzyme in glutaminolysis, and the CTL gene signature (Figure 1C). Comparable trends between glutamine metabolism markers and individual markers of CTL expansion and effector function (GZMA, GZMB, PRF1, or IFNG) alone (Supplemental Figure 1A; supplemental material available online with this article; https://doi.org/10.1172/JCI140100DS1) were also observed, suggesting that a strong antithetical relationship exists between glutamine metabolism and cytotoxic factors in BLBC tumors.

To evaluate whether glutamine metabolism and CTL activity may influence TNBC patient survival, we stratified BLBC patients based on their tumor glutamine metabolism gene signature (GMGS) as well as their CTL gene signatures (Figure 1D). Patients were then classified into 4 categories: $\mathrm{GMGS}^{\mathrm{LOW}} / \mathrm{CTL}^{\mathrm{HIGH}}$, $\mathrm{GMGS}^{\mathrm{LOW}} / \mathrm{CTL}^{\mathrm{LOW}}, \mathrm{GMGS}^{\mathrm{HIGH}} / \mathrm{CTL}^{\mathrm{HIGH}}$, or $\mathrm{GMGS}^{\mathrm{HIGH}} / \mathrm{CTL}^{\mathrm{LOW}}$, and a Kaplan-Meier plot was used to display patient survival for each group (Figure 1D). Having high expression of glutamine metabolic genes combined with low CTL expression resulted in reduced overall survival as well as distant metastasis-free survival compared with other groups (Figure 1D and Supplemental Figure 1B). Furthermore, survival was significantly reduced when only assessing CTL gene expression with GLS alone (Supplemental Figure 1C). Together, these data suggest that high glutamine metabolism along with low cytotoxic potential of $\mathrm{T}$ cells within tumors may contribute to poorer outcomes in TNBC patients.

Loss of GLS in tumor cells reduces EO771 tumor growth and metastasis in a lymphocyte-dependent manner. Given that human TNBC cells have increased GLS and glutaminolysis and that T cells require glutamine metabolism for proper activation and function (17-19), we assessed the impact of cancer cell-specific glutamine metabolism on T lymphocytes within the tumor microenvironment using a mouse TNBC model. We used CRISPRCas9 technology in the murine TNBC line $\operatorname{E0771}(33,34)$ to generate clonal variants with GLS loss-of-function mutations (Supplemental Figure 2A), referred to here as E0771-GLS ${ }^{\mathrm{KO}}$. Intracellular glutamate, a measure of glutaminolysis, was significantly decreased in the E0771-GLS ${ }^{\mathrm{KO}}$ clones (Supplemental Figure 2B), confirming decreased glutaminolysis in the E0771-GLS ${ }^{\mathrm{KO}}$ cells. Next, wild-type (WT) or GLS ${ }^{\mathrm{KO}}$ cells were orthotopically implanted into the inguinal mammary fat pads of female C57BL/6 mice. Tumors were detected in $100 \%$ of mice from each group; however, tumor growth was diminished substantially in E0771-GLS ${ }^{\mathrm{KO}}$ tumors, as measured by tumor volume (Figure 2A) and tumor weight (Figure 2B). Immunohistochemical (IHC) analysis of tumors identified abundant GLS expression in E0771-WT tumors but not in E0771-GLS ${ }^{\mathrm{KO}}$ tumors (Figure 2C). Ki67 was used as a measure of tumor cell proliferation, and despite the smaller size of E0771-GLS ${ }^{\mathrm{KO}}$ tumors, Ki67 was detected at similar proportions in E0771-GLS ${ }^{\mathrm{KO}}$ and E0771-WT tumors (Figure 2D), suggesting that 
A

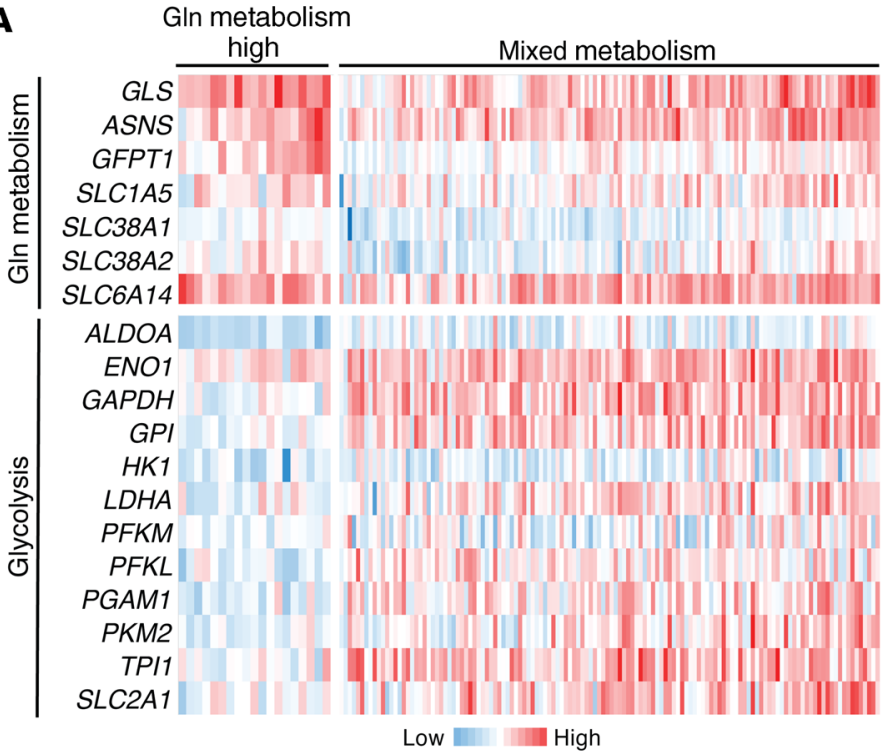

B

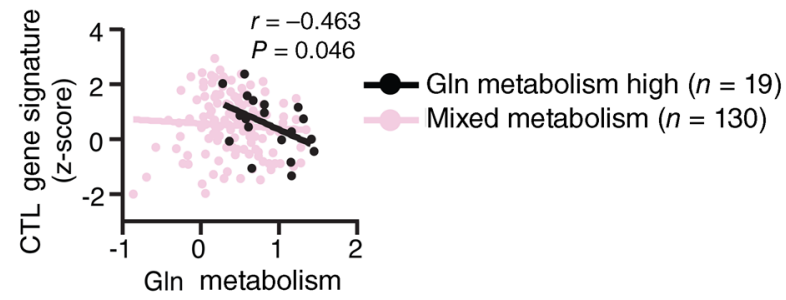

Gene signature (z-score)
C

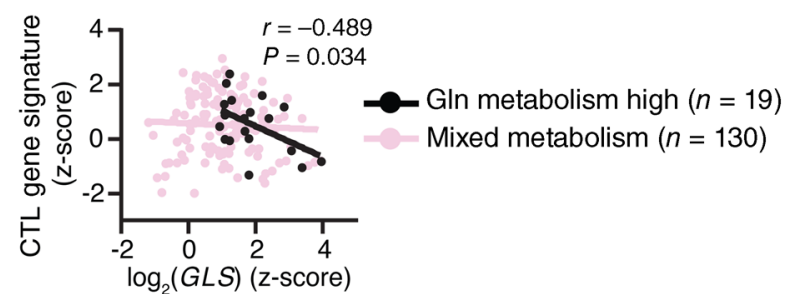

D

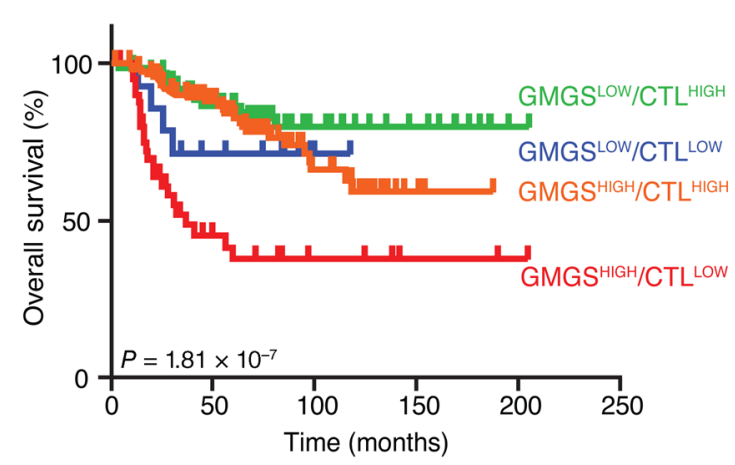

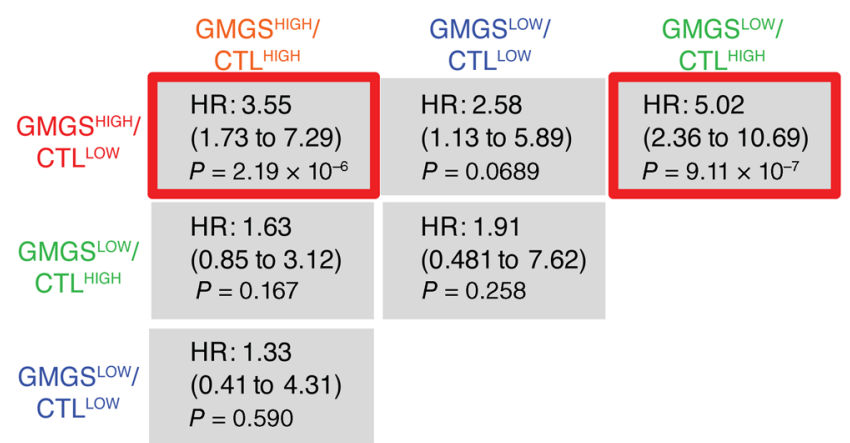

Figure 1. Glutamine metabolism inversely correlates with expression of $\mathrm{T}$ cell activation markers in glutamine metabolism high human basal-like breast cancer samples. (A-C) Analysis of mRNA expression $\left(\log _{2}\right)$ z-scores in basal-like tumor samples from TCCA BRCA data set. The samples with the greatest glutamine metabolism gene signature (GMCS) combined with the lowest glycolysis gene signature are considered "glutamine metabolism high." All others are considered to have "mixed metabolism." (A) Expression levels of individual genes are displayed as a heatmap, with low expression in blue and high expression in red. (B and C) The CTL gene signature ([GZMA + CZMB + PRF1 + INFC]/4) was plotted as a function of the GMCS or GLS for basal-like breast cancer samples that are "glutamine metabolism high" (black) and "mixed metabolism" (pink). Linear regression lines of best fit are shown for both groups. Pearson's correlation analyses are shown. (D) Kaplan-Meier analysis of overall survival of basal breast tumors stratified based on the

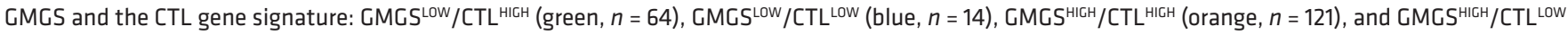
(red, $n=42$ ). Log-rank (Mantel-Cox) test: $P$ values are shown. Red box indicates statistical significance after Bonferroni's correction for multiple comparisons. Hazard ratio (HR) (log-rank) and 95\% confidence intervals are shown.

changes in tumor cell proliferation do not account for diminished tumor growth in the absence of GLS. Further, these data suggest that while E0771 murine TNBC tumor cells import and convert glutamine to glutamate, these tumors are not specifically dependent on glutamine metabolism for their growth in vivo, making these tumors an ideal model for assessing the impact of tumor glutamine metabolism on cells of the tumor microenvironment.

To evaluate the role of tumor-associated lymphocytes in the observed reduction of E0771-GLS ${ }^{\mathrm{KO}}$ tumor growth and metastasis, E0771-WT and E0771-GLS ${ }^{\mathrm{KO}}$ cells were inoculated into the mammary fat pads of Rag1 WT ( $\left.\operatorname{Rag}^{+/+}\right)$or Rag1-deficient $\left(\operatorname{Rag}^{-/-}\right)$ mice, which lack mature $\mathrm{B}$ and $\mathrm{T}$ lymphocytes (35). In Rag1+/+ mice, E0771-GLS ${ }^{\text {KO }}$ tumors grew more slowly than E0771-WT (Figure 2, E and F). However, E0771-GLS ${ }^{\mathrm{KO}}$ tumors grew at a similar rate to E0771-WT tumors when grown in the lymphocyte- devoid microenvironment of Rag1//- mice. Further, IHC for the apoptosis marker cleaved caspase-3 was elevated in E0771-GLS ${ }^{\mathrm{KO}}$ tumors grown in lymphocyte-replete $\mathrm{Ragl}^{+/+}$mice, but not in lymphocyte-devoid Rag1/-- mice (Figure 2G). Similarly, fewer metastatic lung lesions were observed in E0771-GLS ${ }^{\mathrm{KO}}$ tumor-bearing $\mathrm{Ragl}^{+/+}$mice, but this difference was lost in $\mathrm{Rag1}^{-/-}$animals (Figure $2 \mathrm{H}$ and $\mathrm{I}$ ). Together, these data suggest that lymphocytes are necessary to induce tumor cell death and reduce tumor metastasis in E0771-GLS ${ }^{\mathrm{KO}}$ tumors.

Loss of GLS in mammary epithelia reduces tumor latency and growth, while significantly enhancing T cell activation in the C3(1)-TAg transgenic mouse model. To assess the role of TNBC cell glutamine metabolism on multistep tumorigenesis, we used a genetically engineered spontaneous model, C3(1)-TAg, which expresses the SV40 large T antigen (TAg) in mammary epithelial cells of female 
A

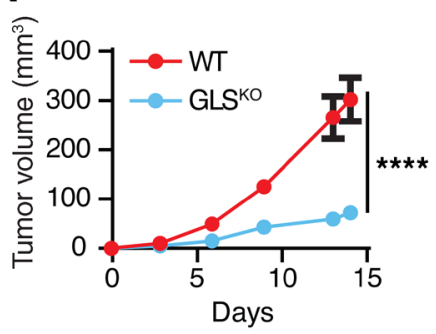

E

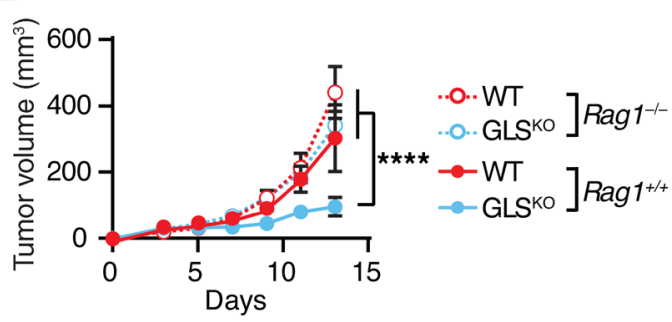

$\mathbf{F}$

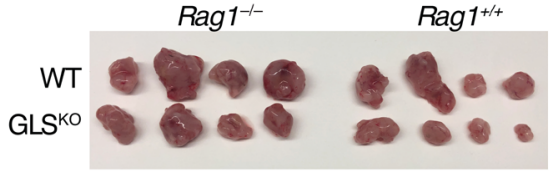

B

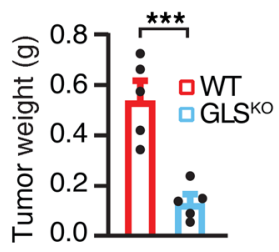

C
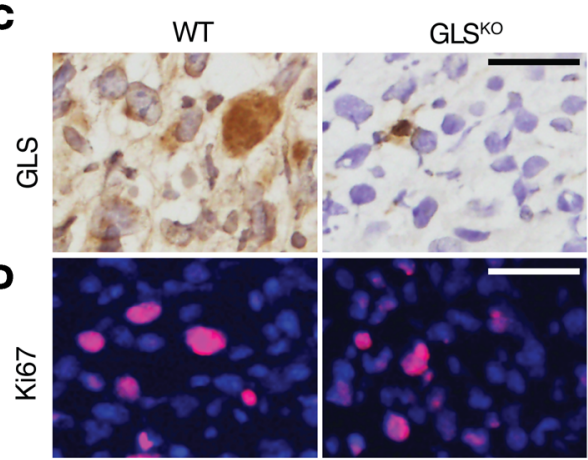

口WT $\square$ GLSKO

G
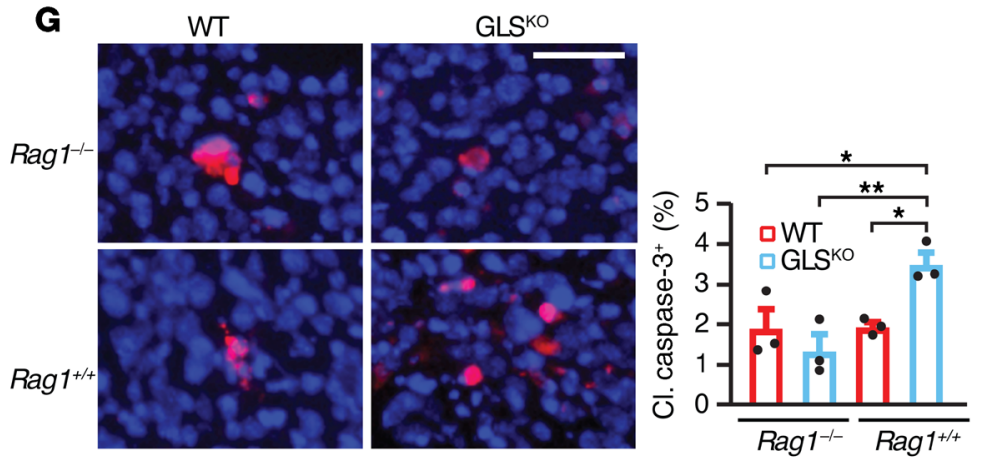

H
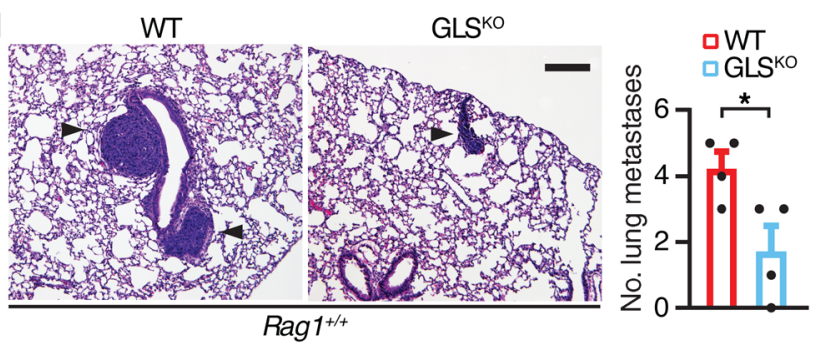
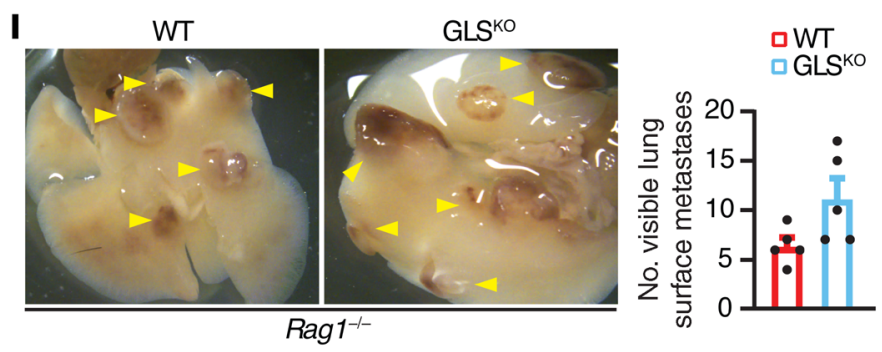

Figure 2. Loss of tumor cell-specific GLS reduces tumor growth and metastasis in a lymphocyte-dependent manner in an orthotopic model of TNBC. (A-D) E0771 sgLacZ (E0771-WT, red) or sgGls_3C15 (E0771-GLSKo, blue) $\left(5 \times 10^{5}\right)$ cells were implanted into the number 4 mammary fat pad of female C57BL/6 mice (Jackson Laboratories), and tumors were harvested after 14 days. (A) Tumor volume $\left(P=1.19 \times 10^{-10}\right.$ by 2-way ANOVA) and (B) tumor weight at harvest $\left(P=7.94 \times 10^{-4}\right.$ by unpaired Student's $t$ test) are shown. $n=5$ mice per group. (C and $\left.\mathbf{D}\right)$ Tumor sections from $\mathbf{A}$ and $\mathbf{B}$ were stained by $(\mathbf{C})$ immunohistochemistry for GLS (brown) or immunofluorescence for (D) Ki67 (red). Nuclei were stained with (C) hematoxylin (blue) or (D) DAPI (blue). Total nuclei and Ki67+ cells were counted from 3 fields of view using Imagel software. Scale bars: $10 \mu \mathrm{m}$ (GLS) and $20 \mu \mathrm{m}$ (Ki67). $P=0.84$ by unpaired Student's $t$ test.

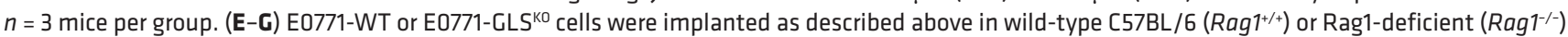
mice. (E) Plot of tumor volumes is shown. $P=2.66 \times 10^{-6}$ by 2-way ANOVA. (F) Image of tumors harvested from $\mathbf{E}(n=4$ mice per group). (C) Immunofluorescence of cleaved caspase-3 (red). Nuclei were stained with DAPI (blue). $P=0.011$ by 1-way ANOVA with Tukey's post hoc analysis for multiple comparisons. $n=3$ mice per group. $\left(\mathbf{H}\right.$ and I) C57BL/6 $\left(\right.$ Rag $\left.^{1 /+}\right)(\mathbf{H})$ or Rag1 $1^{-/-}(\mathbf{I})$ mice were implanted with E0771-WT or E0771-GLS ${ }^{\mathrm{KO}}$ cells $\left(2.5 \times 10^{5}\right)$. (H) Lungs were harvested when tumors reached 400-600 mm (21-27 days). Micrometastases (black arrows) were scored from $3 \mathrm{H \& E}$-stained sections isolated $100 \mu \mathrm{m}$ apart ( $n=4$ mice per group). Scale bar: $200 \mu \mathrm{m}$. (I) Tumors were resected at 1400-1500 mm³ and lungs were harvested between 13 and 20 days after resection ( $n=5$ mice per group). Surface metastases (yellow arrows) were scored from whole lung specimens. Unpaired Student's $t$ test: $(\mathbf{H}) P=0.031$,

(I) $P=0.062 .{ }^{*} P<0.05,{ }^{* *} P<0.01,{ }^{* * *} P<0.005,{ }^{* * * *} P<0.001$.

mice via the promoter for the C3(1) component of the rat prostate steroid binding protein (PSBP) (36). Importantly, genetic profiling studies showed that the C3(1)-TAg mouse model recapitulated BLBC gene signatures seen in human TNBC/BLBC specimens, including an abundance of genes representing proliferation, cell cycle pathways, and chromosome instability gene signatures (37, 38). C3(1)-TAg mice were crossed into genetically engineered mice with floxed $G L S$ alleles $\left(G L S^{f / f l}\right)(26)$ and with transgenic $M M T V$ Cre mice expressing Cre recombinase (39), allowing for Cremediated recombination at floxed GLS alleles in mammary epi- thelial cells (39). While C3(1)-TAg; GLS $S^{f / f l}$ mice lacking MMTV-Cre (referred to here as $T A g$ - $G L S^{f / f l}$ ) developed tumors with an average latency of 34.4 weeks, latency was significantly delayed to 40.2 weeks in C3(1)-TAg; MMTV-Cre; GLS $S^{f / f l}$ (referred to as TAg-GLS $S^{\Delta \Delta}$ ) (Figure 3A). Kaplan-Meier analysis of survival in tumor-bearing mice revealed extended survival in $T A g-G L S^{\Delta \Delta}$ mice as compared with $T A g-G L S^{f / f l}$ (Figure 3B). To rule out the potentially skewing effects of delayed tumor latency on apparent tumor volume measurements as a function of mouse age, we instead set the time of initial tumor palpation as $t=0$, and then assessed tumor volumes 


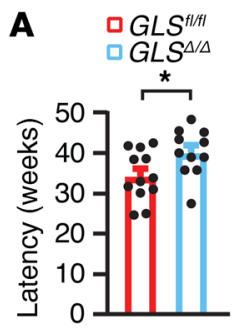

B

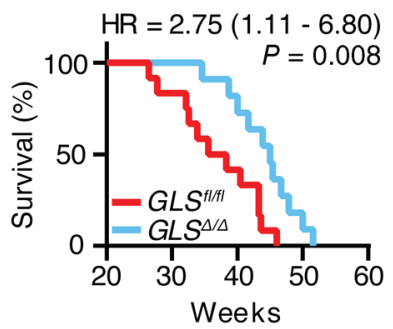

C

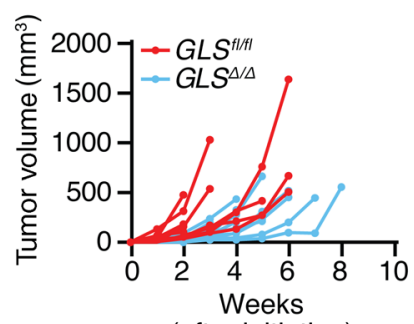

(after initiation)
D

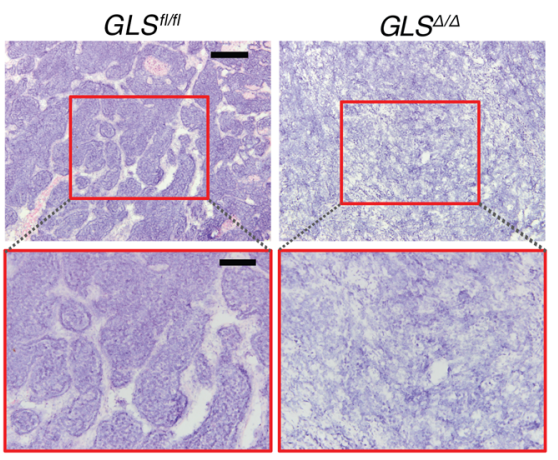

E

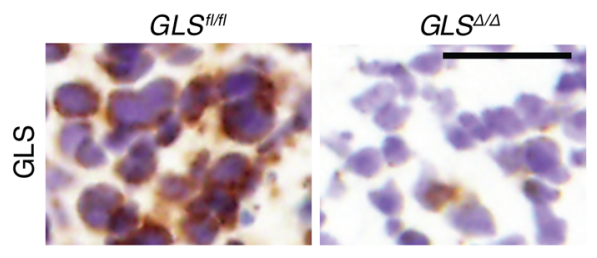

$\mathbf{F}$

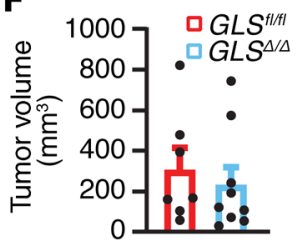

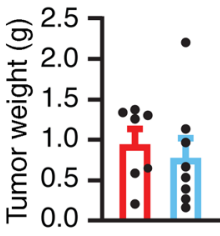

G

2 wks after initiation

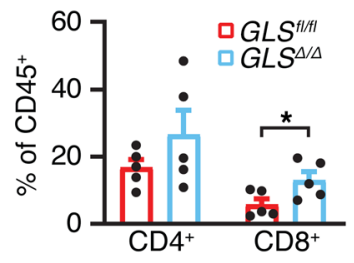

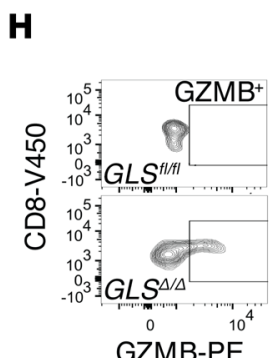

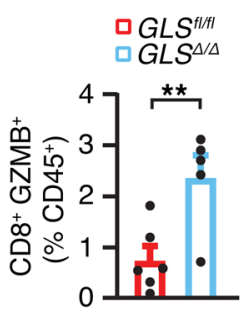

GZMB-PE
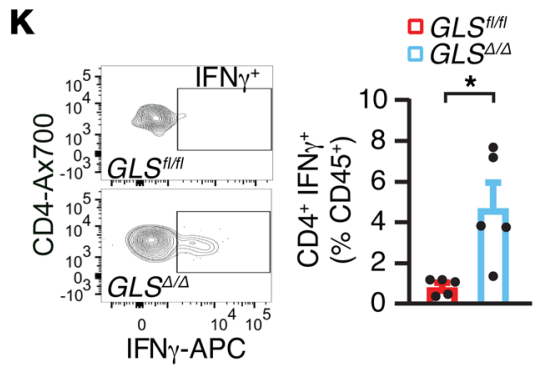
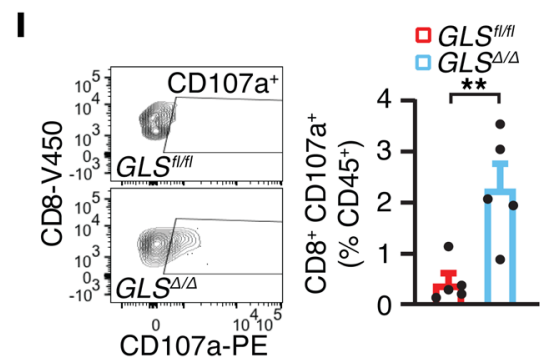

$\mathbf{L} \quad \square G L S^{\prime \prime / I I}$

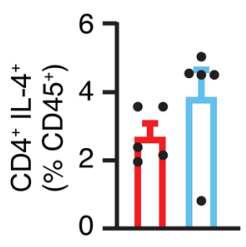

M

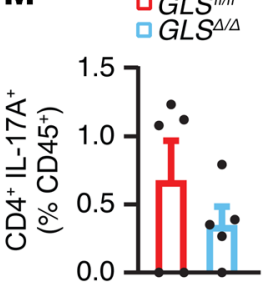

J
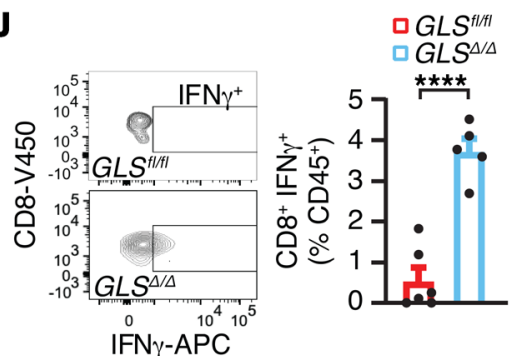

$\mathbf{N}$

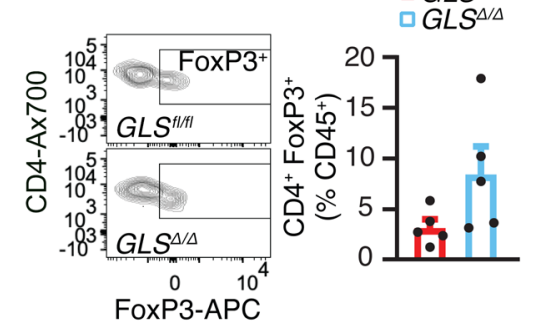

Figure 3. Mammary-specific loss of GLS in a spontaneous TNBC tumor model delays tumor initiation and improves activation of T cells. C3(7)-TAg/CLS fl/fl (GLSfl/fl, red) or C3(1)-TAg; MMTV-Cre; $G L S^{f / / f l}\left(G L S^{\Delta / \Delta}\right.$, blue) mice were palpated weekly for tumor formation and progression. (A) Tumor latency for $G L S^{f / f f l}$ or $C L S^{\Delta / \Delta}$ mice was recorded as the age (weeks) of initial tumor detection. $P=0.031$ by unpaired Student's $t$ test. $n=11-12$ mice per group. (B) Survival (weeks) was determined by the humane endpoint for the $G L S^{f l / f l}$ or $G L S^{\Delta / \Delta}$ from A, plotted as the percentage of surviving mice as a function of age (weeks). $P=0.0082$ by Gehan-Breslow-Wilcoxon test. Hazard ratio was calculated using log-rank analysis, with the $95 \%$ confidence interval shown. (C) Plot of tumor volume after tumor initiation in mice described in A. (D) H\&E images of GLSfl/fl or GLS ${ }^{\Delta / \Delta}$ tumors. Scale bars: $200 \mu \mathrm{m}$ (top) and $100 \mu \mathrm{m}$ (bottom). (E) Immunohistochemistry for GLS (brown) of $G L S^{f l / f l}$ or $G L S^{\Delta / \Delta}$ tumors. Nuclei were stained with hematoxylin (blue). Scale bar: $20 \mu \mathrm{m}$. (F) Tumors were harvested from $G L S^{f l / f l}$ (red) or $G L S^{\Delta / \Delta}$ (blue) mice at 1 to 2 weeks after initial tumor detection. Tumor volume (mm ${ }^{3}$ ) (left) and tumor mass (grams) (right) were recorded at harvest. Unpaired Student's $t$ test: $P=0.581$ (volume), $P=0.581$ (mass). $n=7-9$ mice per group. (G-N) Flow cytometric analyses of whole tumor preparations. $n=5-6$ mice per group. (C) CD4+ (left) or CD8+ (right) T cells, plotted as percentage of CD45+ immune cells. Unpaired Student's $t$ test: $P=0.226\left(\mathrm{CD}^{+}\right), P=0.043\left(\mathrm{CD} 8^{+}\right)$. (H-N) Flow cytometric analyses of (H) CD8 ${ }^{+} \mathrm{CZMB}^{+}$, (I) CD8 $8^{+} \mathrm{CD} 107 \mathrm{a}^{+},(\mathrm{J}) \mathrm{CD} 8^{+} \mathrm{IFN}-\gamma^{+},(\mathrm{K}) \mathrm{CD} 4^{+} \mathrm{IFN}-\gamma^{+},(\mathrm{L}) \mathrm{CD} 4^{+} \mathrm{IL}-4^{+},(\mathrm{M})$ $\mathrm{CD}^{+} \mathrm{IL}-17 \mathrm{~A}^{+}$, and $(\mathbf{N}) \mathrm{CD}^{+} \mathrm{FoxP3}^{+} \mathrm{T}$ cells in $\mathrm{CLS}^{f / f f}$ (red) or $\mathrm{CLS}^{\Delta / \Delta}$ (blue) tumors, plotted as a percentage of CD45+ cells. Unpaired Student's $t$ test: $P=0.0085$

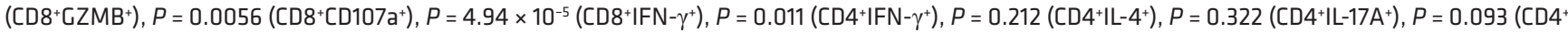
FoxP3 $\left.{ }^{+}\right)$. ${ }^{*} P<0.05,{ }^{* *} P<0.01,{ }^{* * * *} P<0.001$.

in the weeks following initial tumor palpation. This approach revealed markedly reduced growth of $T A g-G L S^{\Delta \Delta}$ tumors as compared with $T A g-G L S^{A / f l}$ (Figure 3C). Histological analysis of tumors collected from mice at humane endpoints revealed dense, high- ly cellular tumors with tightly packed nuclei in $T A g-G L S^{A / f l}$ mice. However, vast central areas lacking tumor cellularity were noted in $T A g$-GLS $S^{\Delta \Delta}$ tumors (Figure 3D). IHC detection of GLS confirmed loss of GLS protein expression in $T A g-G L S^{\Delta \Delta}$ tumors (Figure 3E). 
In order to assess how tumor cell-specific GLS impacts T cells within the tumor microenvironment, $\mathrm{T}$ cell populations were analyzed from tumor single-cell suspensions using flow cytometry (Supplemental Figure 3A). Because tumor size may affect antitumor immunity, we analyzed tumors harvested at 2 weeks after initial tumor palpation, a time point when both groups displayed similar tumor volumes and weights (Figure $3 \mathrm{~F}$ ). TAg-GLS $S^{\Delta / \Delta}$ tumors harbored significantly increased levels of $\mathrm{CD} 45^{+}$immune cells and CD $45^{+} \mathrm{CD}^{+}$TILs (Supplemental Figure 3, B and C), with greater enrichment of $\mathrm{CD}^{+} \mathrm{CD}^{+} \mathrm{T}$ cells (Figure $3 \mathrm{G}$ and Supplemental Figure 3D). Cytotoxic CD ${ }^{+} \mathrm{T}$ cells directly carry out their killing activity via release of the serine protease, granzyme B (GZMB) (40), and consistent with increased effector function, $T A g-G L S^{\Delta / \Delta}$ tumors harbored increased $\mathrm{GZMB}^{+} \mathrm{CD} 8^{+} \mathrm{T}$ cells compared with $T A g-G L S^{f / f l}$ controls (Figure $3 \mathrm{H}$ and Supplemental Figure 3E). TAg$G L S^{\Delta / \Delta}$ tumors also displayed increased cytotoxic T lymphocytes (CTLs) expressing the degranulation marker CD107a (Figure 3I and Supplemental Figure 3F), the proinflammatory cytokine interferon $\gamma($ IFN- $\gamma$ ) (Figure 3J, and Supplemental Figure 3G), as well as the cell activation markers CD25 and CD44 (Supplemental Figure $3, \mathrm{H}-\mathrm{J})$. Tumor cell loss of GLS also resulted in a modest increase in both the number of IFN- $\gamma$-producing CD4 ${ }^{+}$Th1 T helper cells and the IFN- $\gamma$ median fluorescence intensity (Figure 3K and Supplemental Figure 3K). Smaller increases were observed in the $\mathrm{IL}-4^{+} \mathrm{Th} 2$ as well as the FoxP $3^{+}$and FoxP $3^{+} \mathrm{CD} 25^{+}$Treg $\mathrm{CD} 4^{+} \mathrm{T}$ cell populations in $T A g-G L S^{\Delta / \Delta}$ tumors, whereas a small decrease in the IL-17A ${ }^{+}$Th17 population was detected, although these changes were not associated with increased cytokine production or FoxP3 expression (Figure 3, L-N, and Supplemental Figure 3, L-Q). Interestingly, the proportion of $\mathrm{CD}^{+} \mathrm{T}$ cells expressing activation markers CD25, CD69, and CD44 was largely unchanged, suggesting that cumulative $\mathrm{CD} 4^{+} \mathrm{T}$ cell activation may not be occurring in $T A g-G L S^{\Delta / \Delta}$ tumors (Supplemental Figure 3, R-T). Together, these findings suggest that targeting tumor-specific GLS improves $\mathrm{CD}^{+}$ T cell activation and effector capacity with this TNBC model.

Tumor cell GLS deficiency in established tumors increases $T$ cell numbers and effector function. T cell numbers and activation markers were also examined in orthotopic E0771-WT and E0771-GLS ${ }^{\mathrm{KO}}$ tumors grown in WT C57BL/6 mice. Flow cytometric tumor analyses identified increased numbers of $\mathrm{CD}_{4} 5^{+}$tumor-associated leukocytes and $\mathrm{CD}_{4} 5^{+} \mathrm{CD}^{+} \mathrm{T}$ cells in E0771-GLS ${ }^{\mathrm{KO}}$ tumors as compared with E0771-WT (Supplemental Figure 4, A and B). The percentage of the $\mathrm{CD} 45^{+}$population that was also $\mathrm{CD}^{+} \mathrm{CD} 8^{+}$was increased in E0771-GLS ${ }^{\mathrm{KO}}$ tumors compared with the WT controls (Figure 4A). A trend toward an increased percentage of CD45 tumor leukocytes that were $\mathrm{CD} 4^{+}$was also seen in E0771-GLS ${ }^{\mathrm{KO}}$ tumors. When normalized to total tumor mass, the numbers of both $\mathrm{CD}^{+}$and $\mathrm{CD}^{+} \mathrm{T}$ cells were increased in E0771-GLS ${ }^{\mathrm{KO}}$ tumors as compared with controls (Figure 4B).

Further, $\mathrm{CD}^{+} \mathrm{T}$ cells expressing mature CTL effector markers were increased in E0771-GLS ${ }^{\mathrm{KO}}$ tumors, including those expressing GZMB (Figure 4C), CD107a (Figure 4D), and IFN- $\gamma$ (Figure $4 \mathrm{E}$ ), while CTL activation markers were also increased, including CD25, CD69, and CD44, (Supplemental Figure 4, C-E), suggesting that GLS loss from the tumor epithelia may indirectly enhance the differentiation and effector activity of $\mathrm{CD}^{+} \mathrm{T}$ cells. E0771-GLS ${ }^{\mathrm{KO}}$ tumors also exhibited an increased presence of $\mathrm{CD} 4^{+} \mathrm{IFN}-\gamma^{+} \mathrm{Th} 1$ cells (Figure $4 \mathrm{~F}$ ), although the number of the CD $4^{+} \mathrm{IL}-4^{+}$Th2 cells was not significantly altered by GLS loss from tumor cells (Figure $4 \mathrm{G}$ ). Interestingly, CD4 $4^{+} \mathrm{IL}-17 \mathrm{~A}^{+} \mathrm{Th} 17$ populations were also increased in E0771-GLS ${ }^{\mathrm{KO}}$ tumors (Figure $4 \mathrm{H}$ and Supplemental Figure 4F), although no significant changes were observed in Tregs (Figure 4I and Supplemental Figure 4F). Additionally, the number of $\mathrm{CD}^{+}{ }^{+} \mathrm{T}$ cells from E0771-GLS ${ }^{\mathrm{KO}}$ tumors expressing the activation markers CD25, CD69, and CD44 was increased, consistent with increased $\mathrm{CD} 4^{+} \mathrm{T}$ cell activation (Supplemental Figure 4, G-I). Collectively, these data suggest that deletion of GLS in TNBC tumor cells enhances the number and effector function of tumor-infiltrating T cells, possibly through changes in the metabolite availability within the tumor microenvironment in which these lymphocytes must function.

Loss of GLS in tumor cells increases interstitial glutamine and enhances antigen-specific antitumor $C D 8^{+} T$ cell infiltration. Glutamine is an abundant amino acid in the blood stream, but glutamine supply can vary in the tissue interstitium $(41,42)$. Notably, glutamine concentrations measured within tumor interstitial fluid collected from E0771-WT tumors was approximately $100 \mu \mathrm{M}$, whereas E0771-GLS ${ }^{\text {KO }}$ interstitial tumor glutamine levels were nearly $700 \mu \mathrm{M}$ (Figure 4J), similar to the reported plasma glutamine concentration of C57BL/6 mice $(41,43)$. These data suggest that GLS loss from tumor cells prevents tumor cell-driven glutamine depletion within the interstitium, which may provide more available glutamine for other cells in the tumor microenvironment, including lymphocytes. Indeed, we observe that IFN- $\gamma$ secretion from effector type I CD $8^{+} \mathrm{T}$ cells (Tc1) was significantly increased in response to elevated glutamine (Figure $4 \mathrm{~K}$ ), suggesting that greater glutamine availability in $\mathrm{GLS}^{\mathrm{KO}}$ tumor interstitium may directly improve CTL effector responses.

To analyze how tumor cell glutamine metabolism may regulate an antigen-specific T cell response, we utilized E0771(OVA)WT and E0771(OVA)-GLS ${ }^{\text {KO }}$ cells expressing the full-length ovalbumin (OVA) protein (Supplemental Figure 4, J and K). OVA peptides are processed for presentation of the $\mathrm{OVA}_{257-264}$ antigenic peptide (SIINFEKL) on MHC class I (H2K ${ }^{b}$ ) molecules (44). CD8 ${ }^{+}$ $\mathrm{T}$ cells expressing a SIINFEKL-specific $\mathrm{T}$ cell receptor (TCR) $(45,46)$ were purified from OT-I transgenic mice (Supplemental Figure 4L), transduced ex vivo to overexpress GFP, and then primed with SIINFEKL peptide. Following E0771(OVA) tumor engraftment, adoptive transfer of $\mathrm{OT}-\mathrm{I} \mathrm{GFP}^{+} \mathrm{CD}^{+} \mathrm{T}$ cells into tumor-bearing mice enabled immunofluorescent visualization of CTL tumor infiltration (Figure $4 \mathrm{~L}$ ). Although OT-I GFP $\mathrm{CD}^{+} \mathrm{T}$ cells were detected in all tumors, $\mathrm{GFP}^{+}$cells were more abundant in E0771(OVA)-GLS ${ }^{\mathrm{KO}}$ tumors (Figure 4M). Flow cytometry confirmed this observation, revealing nearly twice as many $\mathrm{GFP}^{+} \mathrm{CD}^{+}$ T cells from E0771-GLS ${ }^{\mathrm{KO}}$ tumors as compared with E0771-WT tumors (Figure $4 \mathrm{~N}$ ). Together, these results suggest that inhibition of glutamine utilization within tumor cells increases the intratumoral $\mathrm{CD}^{+} \mathrm{T}$ cells.

Glutamine transporter inhibition by $V$-9302 reduces tumor growth and improves $T$ cell activation. Because genetic models of impaired tumor cell glutaminolysis resulted in decreased tumor growth, increased glutamine concentrations in the tumor interstitium, and improved TIL numbers, we tested the hypothesis that pharmacologic blockade of glutamine metabolism might significantly 
A

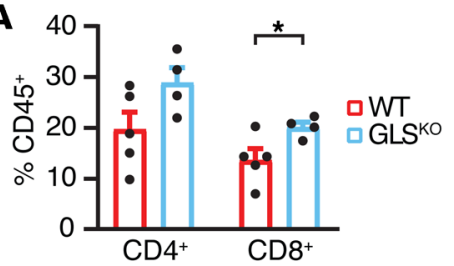

B

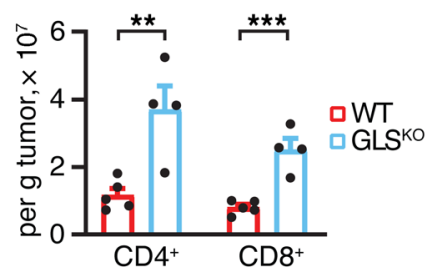

C

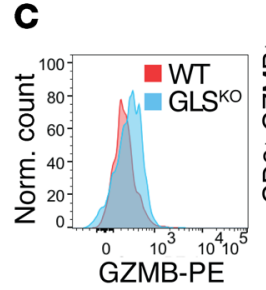

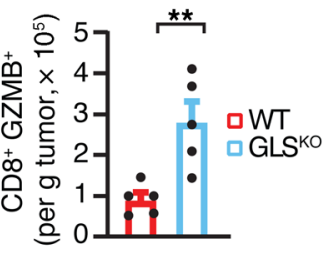

D

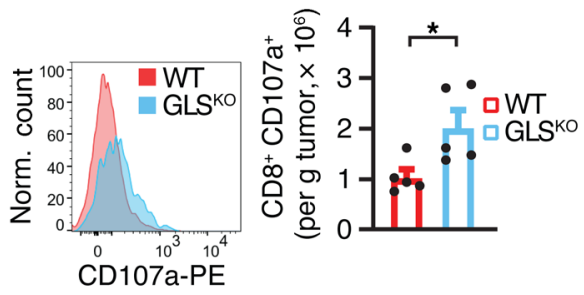

$\mathbf{F}$

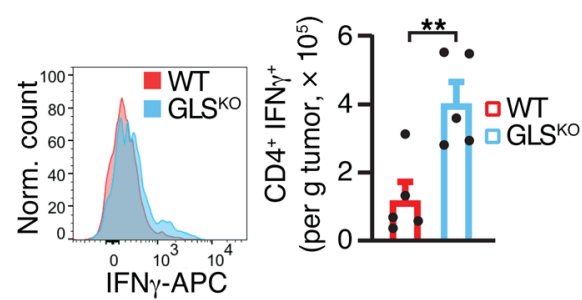

H

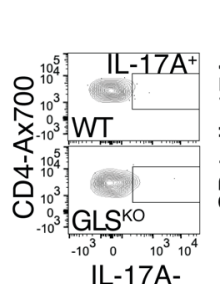

PerCP-Cy5.5
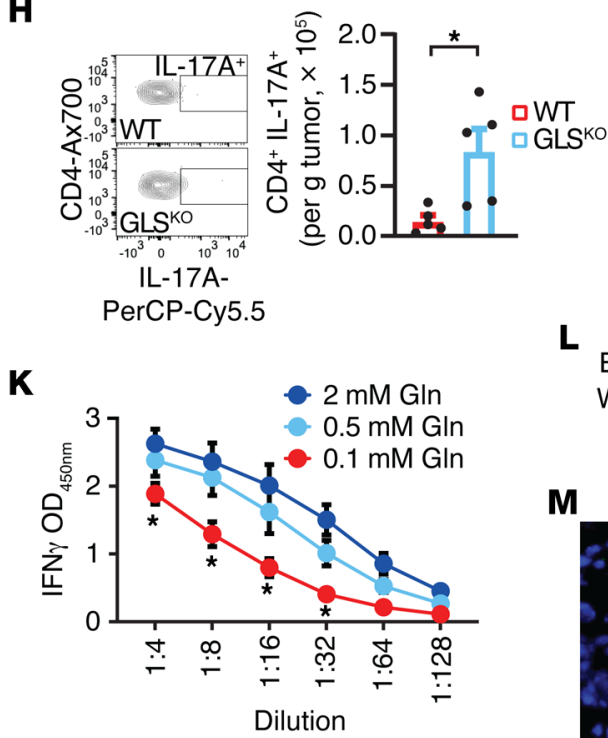

L
E
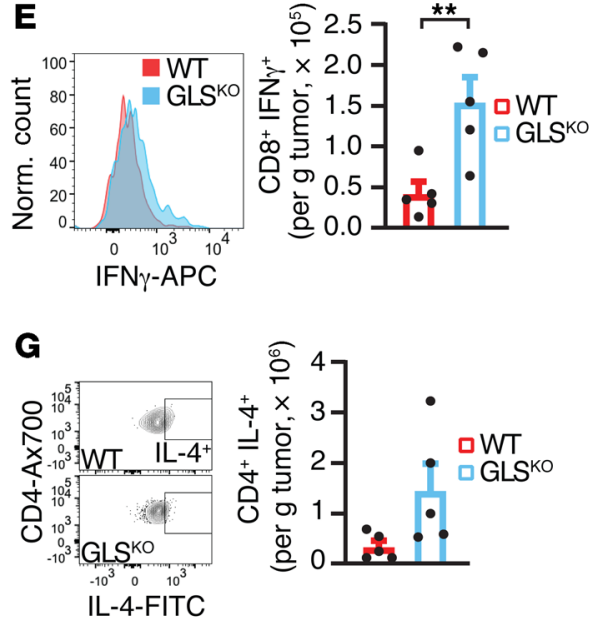

I

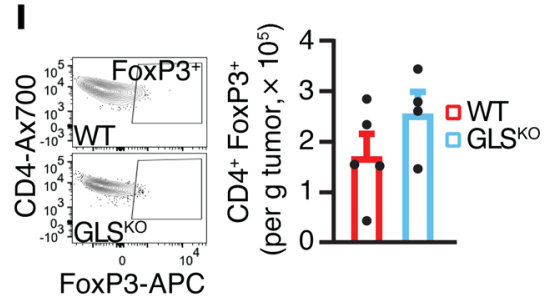

FoxP3-APC
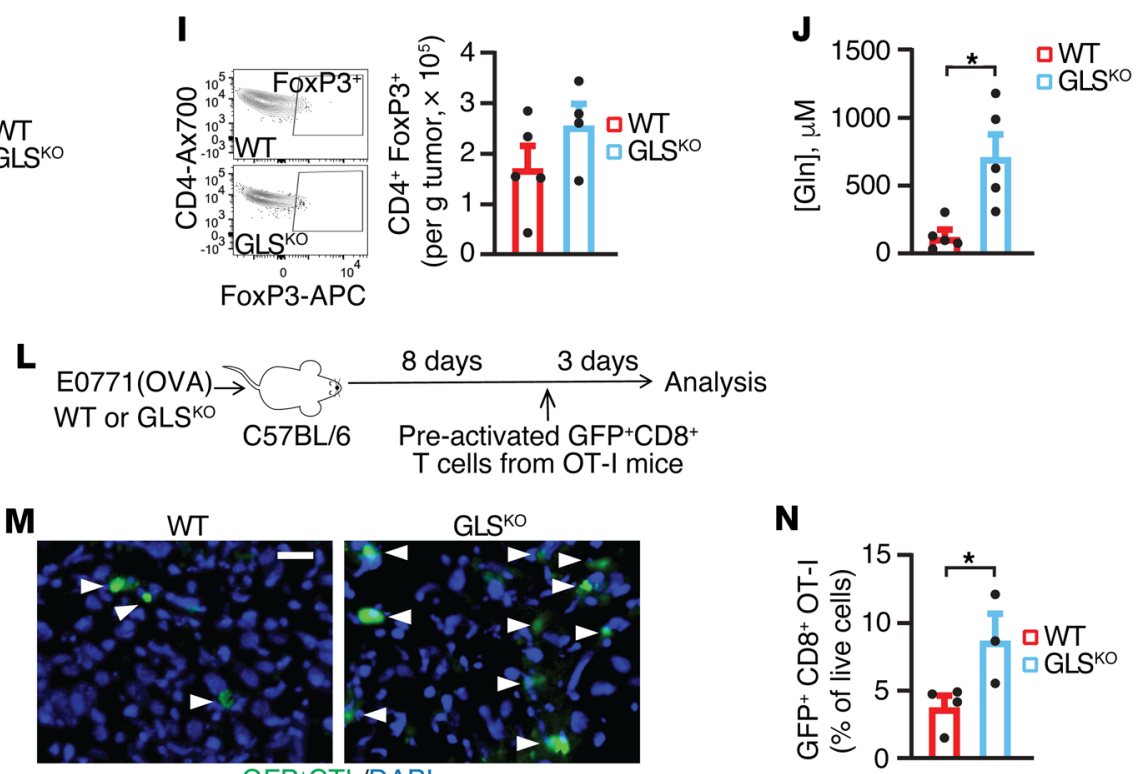

Figure 4. Loss of tumor cell-specific GLS increases T cell activation in an orthotopic TNBC tumor model. (A-I) E0771 sgLacZ (WT, red) or sgGls_3C15 (GLS ${ }^{\mathrm{K}}$, blue) $\left(5 \times 10^{5}\right)$ cells were implanted into the number 4 mammary fat pad of female C57BL/6 mice (Jackson Laboratories). Tumors were harvested after 14 days. $n=4-5$ mice per group. (A and B) Flow cytometric analyses of CD4+ (left) or CD8 ${ }^{+}$(right) T cells, plotted as percentage of (A) CD45 immune cells or (B) total cells normalized to tumor mass (gram). Unpaired Student's $t$ test: $\mathrm{CD}^{+},(\mathbf{A}) P=0.091$, (B) $P=0.0063 ; \mathrm{CD}^{+},(\mathbf{A}) P=0.040,(\mathbf{B}) P=8.23$ $\times 10^{-4}$. (C-I) Flow cytometric analyses of tumors for (C) CD8+CZMB', (D) CD8+CD107a+, (E) CD8 $8^{+}$IFN- $\gamma^{+},(\mathbf{F}) \mathrm{CD}^{+} \mathrm{IFN}-\gamma^{+},(\mathbf{C}) \mathrm{CD}^{+} \mathrm{IL}-4^{+},(\mathbf{H}) \mathrm{CD}^{+} \mathrm{IL}-17 \mathrm{~A}^{+}, \mathrm{and}^{+}$ (I) $\mathrm{CD}^{+}{ }^{+} \mathrm{FoxP3}^{+} \mathrm{CD}^{+} 5^{+} \mathrm{CD}^{+} \mathrm{T}$ cells. Total counts were normalized per tumor mass (grams). Unpaired Student's $t$ test: $P=0.0064$ (CD8 $\left.{ }^{+} \mathrm{CZMB} \mathrm{B}^{+}\right), P=0.027$

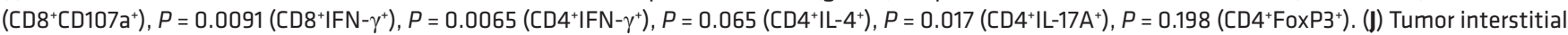
fluid was collected from E0771-WT (red) or E0771-GLSKO (blue) tumors via centrifugation of harvested tumors. Glutamine (gIn) was measured and concentration $(\mu \mathrm{M})$ was calculated against a glutamine standard. $P=0.021$ by paired Student's $t$ test. $n=5$ mice per group. (K) IFN- $\gamma$ ELISA of supernatants collected from Tc1 CD8 ${ }^{+}$T cells activated (anti-CD3/anti-CD28) in media supplemented with 0.1, 0.5, or 2 mM glutamine $(n=3)$. Two-way ANOVA; unpaired Student's $t$ test. (L-N) E0771(OVA) sgLacZ (WT, red) or sgGls3 (GLS ${ }^{\mathrm{K} 0}$, blue) $\left(5 \times 10^{5}\right)$ cells were bilaterally implanted into the number 4 mammary fat pads

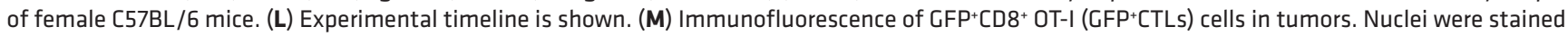
with DAPI (blue). Arrows indicate GFP+CD8 ${ }^{+}$OT-I T cells. Scale bar: $20 \mu \mathrm{m}$. (N) Flow cytometric analysis of GFP+CD8 ${ }^{+}$T cells from whole cell tumor preparations, plotted as percentage of all live cells. $P=0.043$ by unpaired Student's $t$ test. $n=3-4$ mice per group. ${ }^{*} P<0.05,{ }^{* *} P<0.01,{ }^{* * *} P<0.005$. 
diminish growth and support T cell activity in TNBC models. Early clinical studies of the GLS inhibitor CB-839 demonstrated tolerability and safety, but with limited efficacy as a single agent in TNBC (47). However, V-9302, a small molecule amino acid transporter inhibitor with a greater selectivity for reducing glutamine uptake $(48,49)$, was more efficacious for inducing TNBC cell death in cell culture models, including the human TNBC lines MDA-MB-231 and HCC1806 and the mouse TNBC lines 4T1 and E0771 (Supplemental Figure 5, A-D). Furthermore, V-9302 reportedly has little to no negative impact on CD44 expression on $\mathrm{CD} 8^{+} \mathrm{T}$ cells ex vivo (48). To test the impact of glutamine uptake inhibition with V-9302, orthotopic E0771 tumors grown in immune-competent C57BL/6 female mice were treated daily with V-9302 $(50 \mathrm{mg} / \mathrm{kg})$ or vehicle beginning when tumors reached $100 \mathrm{~mm}^{3}$, equivalent to 11 days after tumor cell inoculation. Tumors treated with V-9302 displayed markedly reduced tumor growth (Figure 5A), resulting in decreased tumor weight upon collection on day 16, after only 5 days of treatment (Figure 5B). While V-9302 had only a marginal impact on $\mathrm{Ki}^{+} 7^{+}$cell proliferation, there was a more significant (3-fold) increase in apoptosis, as measured by cleaved caspase-3 (Figure 5C), in agreement with the increased cell death seen upon genetic GLS loss in E0771 tumors.

Interestingly, the number of $\mathrm{CD} 45^{+}$leukocytes was not significantly impacted by 5 days of V-9302 treatment (Supplemental Figure 5E), nor were the total number of $\mathrm{CD}^{2} 5^{+} \mathrm{CD} 3^{+} \mathrm{T}$ cells (Supplemental Figure 5F). While total $\mathrm{CD}^{+} \mathrm{T}$ cell frequencies were unaffected by V-9302 treatment (Figure 5D), detailed histological assessment identified $\mathrm{CD}^{+} \mathrm{T}$ cells as being restricted to the periphery of vehicle-treated E0771 tumors, while an increased $\mathrm{CD}^{+} \mathrm{T}$ cell infiltration deeper into the tumor core $(>500 \mu \mathrm{m})$ was seen in tumors treated with V-9302 (Figure 5E). Changes in localization from the periphery to the intratumoral core have been described previously, correlating with immune response to therapeutic immune checkpoint inhibition in several tumor models (50-52). We next assessed how V-9302 impacted CD8 ${ }^{+}$TIL activation and effector markers. Tumors treated with V-9302 harbored CD8 ${ }^{+} \mathrm{T}$ cell populations producing GZMB (Figure 5F), CD107a (Figure 5G), IFN- $\gamma$ (Figure 5H), CD25, CD69, and CD44 (Supplemental Figure 5, G and H), confirming CTL effector capacity and activation in response to V-9302. Additionally, CD4 ${ }^{+}$TILs from tumors treated with V-9302 displayed an increased population of IFN- $\gamma$-producing cells, suggesting an increased $\mathrm{CD}^{+}$Th1 $\mathrm{T}$ cell phenotype upon $\mathrm{V}-9302$ treatment (Figure 5I). Only smaller increases in the Th2 (IL-4) and Th17 (IL-17A) populations were observed (Figure 5J and Supplemental Figure 5I). In contrast, the $\mathrm{CD} 4^{+} \mathrm{FoxP} 3^{+} \mathrm{CD} 25^{+} \mathrm{CD} 127^{\text {lo }}$ Treg population was significantly decreased in V-9302-treated tumors, although the FoxP3 ${ }^{+}$population was not changed (Figure $5 \mathrm{~K}$ and Supplemental Figure 5J). The frequency of $\mathrm{CD} 4^{+} \mathrm{T}$ cell populations expressing the activation markers CD25, CD69, and CD44 were also increased (Supplemental Figure 5, K and L). Together, these data confirm that V-9302 increases tumor cell death while simultaneously augmenting $\mathrm{CD} 8^{+} \mathrm{CTL}$ and $\mathrm{CD} 4^{+} \mathrm{Th} 1$ phenotypes within the TNBC tumor microenvironment.

Compared with effector memory (Tem) or terminally differentiated effector $\mathrm{T}$ cells, $\mathrm{CD} 8^{+}$central memory $\mathrm{T}$ cells $(\mathrm{Tcm})$ support a more persistent antitumor response $(53,54)$. To assess whether V-9302 may support long-term cytotoxicity, we evaluated the $\mathrm{CD} 44^{+} \mathrm{CD} 62 \mathrm{~L}^{+} \mathrm{Tcm}$ and $\mathrm{CD} 44^{+} \mathrm{CD} 62 \mathrm{~L}^{-}$Tem populations. Treatment of ex vivo-activated splenocytes with V-9302 significantly increased the Tcm/Tem ratio of $\mathrm{CD}^{+} \mathrm{T}$ cells but not $\mathrm{CD}^{+} \mathrm{T}$ cells (Supplemental Figure 5, M and N). However, both the Tcm and Tem populations were increased in V-9302-treated tumors (Supplemental Figure 5, O and P), suggesting that V-9302 improves antitumor responses in part through supporting persistent memory T cells.

The glutamine transporter inhibitor V-9302 increases glutamine uptake and glutathione synthesis in CTLs. To investigate the underlying molecular mechanisms regulating increased $\mathrm{T}$ cell activation upon treatment with V-9302, splenocytes harvested from WT mice and cultured ex vivo were primed with anti-CD3 and antiCD28 antibodies in the presence or absence of V-9302 $(10 \mu \mathrm{M}$ for 48 hours). As expected, anti-CD3/anti-CD28 priming increased the viable percentage of the total $\mathrm{CD}^{+} \mathrm{T}$ cell population, as compared with splenocytes not primed with anti-CD3/anti-CD28 (Supplemental Figure 6A). Importantly, V-9302 did not affect the proportion of viable $\mathrm{CD}^{+} \mathrm{T}$ cells following anti-CD3/anti-CD28 priming (Supplemental Figure 6A), nor did V-9302 affect the viable $\mathrm{CD}^{+} \mathrm{T}$ cell population (Supplemental Figure 6B). Previous studies demonstrate that anti-CD3/anti-CD28 priming of $\mathrm{T}$ cells activates the mTORC1 signaling pathway, resulting in phosphorylation of the mTORC1 effector ribosomal protein S6 (S6RP) (55, 56). As measured by phos-flow cytometry, anti-CD3/anti-CD28 priming of $\mathrm{CD}^{+} \mathrm{T}$ cells increased phospho-S6RP, which was unaffected by V-9302 (Supplemental Figure 6C). Similarly, V-9302 did not significantly alter phospho-S6RP in $\mathrm{CD}^{+} \mathrm{T}$ cells (Supplemental Figure 6D). The cytotoxic capabilities of OT-I CD8 ${ }^{+} \mathrm{T}$ cells against E0771(OVA) tumor cells were assessed in coculture studies performed in the presence and absence of V-9302. Importantly, OT-I CD8 ${ }^{+} \mathrm{T}$ cells were poorly cytotoxic against parental E0771 cells, but displayed potent cytotoxicity against E0771(OVA) cells, confirming antigen-directed cytotoxicity against tumors cells (Figure 6A). Notably, treatment with V-9302 increased T celldependent cytotoxicity in this assay nearly 2 -fold, indicating that $\mathrm{V}-9302$ enhances the ability of $\mathrm{CD}^{+} \mathrm{T}$ lymphocytes to kill tumor cells. These studies suggest that V-9302 enhances the cytotoxicity of $\mathrm{CD}^{+} \mathrm{T}$ cells but has no significant impact on mTORC1 activation or T cell viability upon TCR stimulation, despite the well-documented requirement for glutamine uptake and glutaminolysis in $\mathrm{T}$ cell viability and activation.

Still, similar to our observation in a GLS deletion model, $\mathrm{V}-9302$ increased the tumor interstitial glutamine concentration (Figure 6B), indicating that V-9302 may improve glutamine availability for $\mathrm{T}$ cells. These observations were assessed further by measuring ${ }^{3} \mathrm{H}$-glutamine uptake in anti-CD3/anti-CD28-primed $\mathrm{CD}^{+} \mathrm{T}$ cells cocultured with E0771 tumor cells. After pulsing for 15 minutes with ${ }^{3} \mathrm{H}$-glutamine, tumor cells and $\mathrm{CD} 8^{+} \mathrm{T}$ cells were assessed separately for ${ }^{3} \mathrm{H}$-glutamine uptake. While ${ }^{3} \mathrm{H}$-glutamine was detected in E0771 cells upon coculture with $\mathrm{CD}^{+} \mathrm{T}$ cells, treatment of cocultures with $\mathrm{V}-9302$ diminished ${ }^{3} \mathrm{H}$-glutamine uptake by E0771 tumors nearly 40\% (Figure 6C). In contrast, V-9302 treatment did not diminish ${ }^{3} \mathrm{H}$-glutamine uptake by $\mathrm{CD}^{+}$ $\mathrm{T}$ cells, and even increased $\mathrm{CD} 8^{+} \mathrm{T}$ cell ${ }^{3} \mathrm{H}$-glutamine uptake, a differential effect that was also seen in isolated cells (Supplemen- 
A

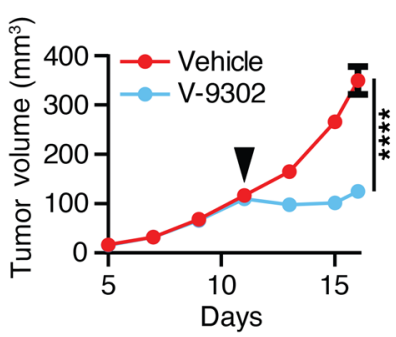

B

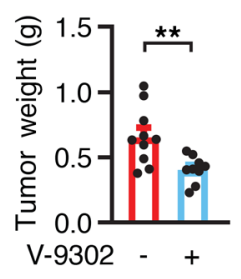

C
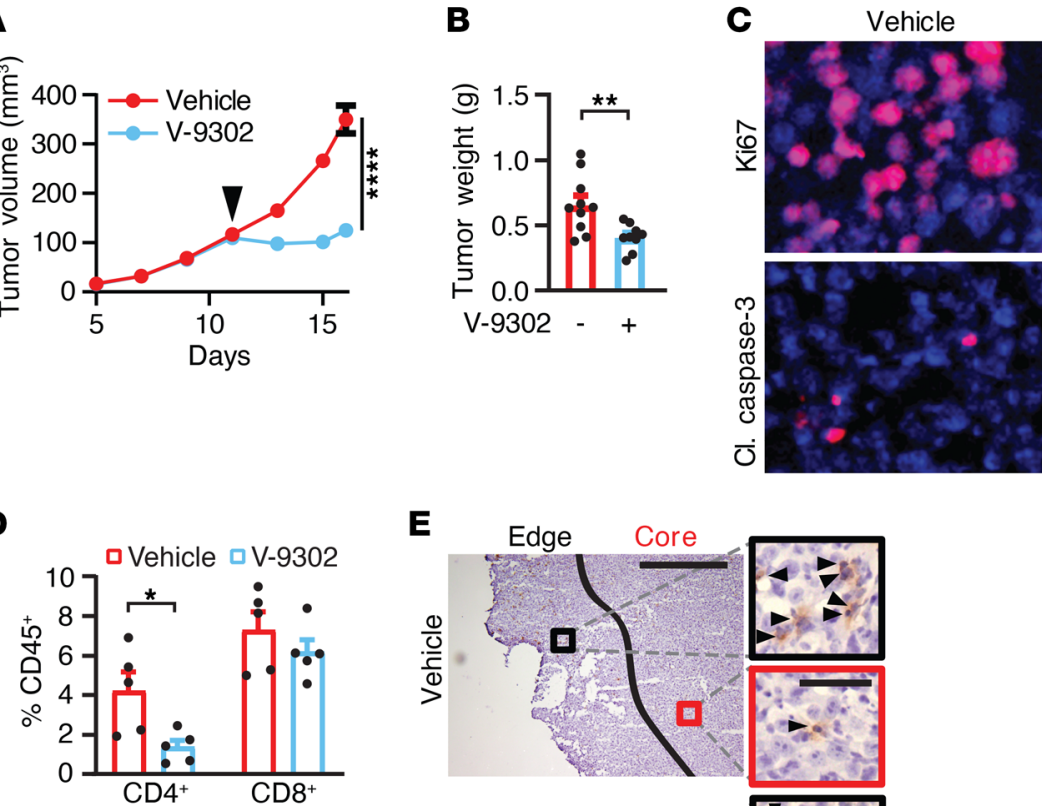

E

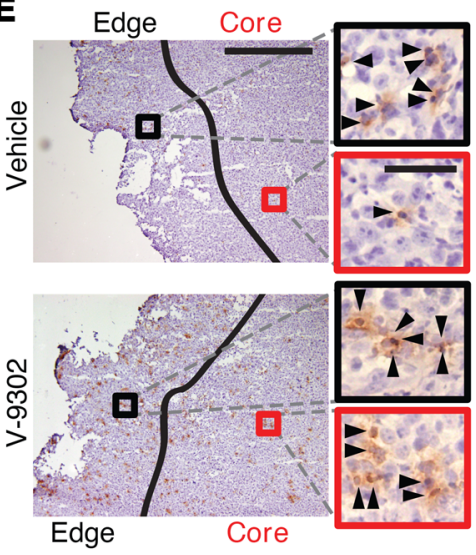

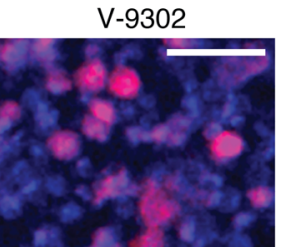

व Veh $\square$ V-9302
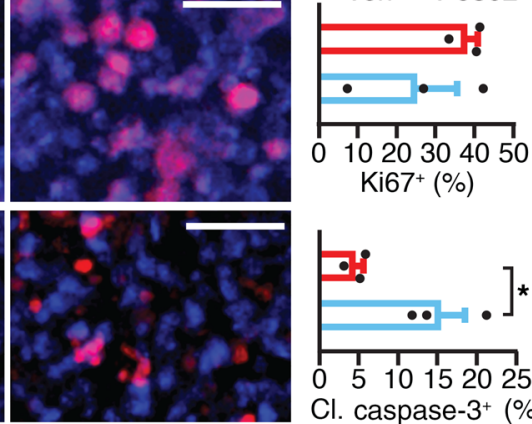

Cl. caspase- $3^{+}(\%)$
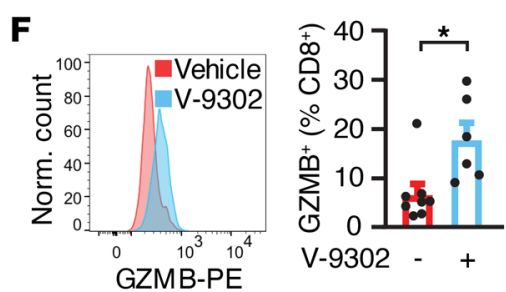

G
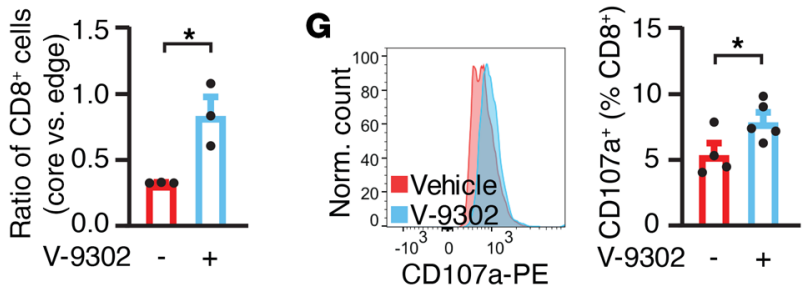

H
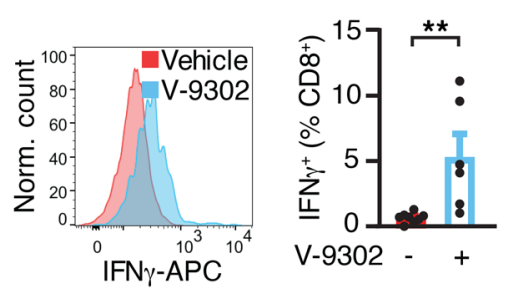
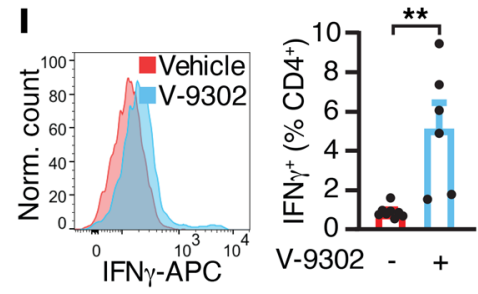

J

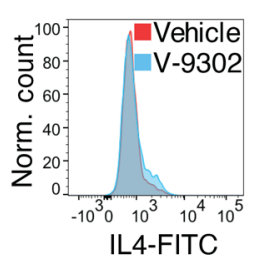

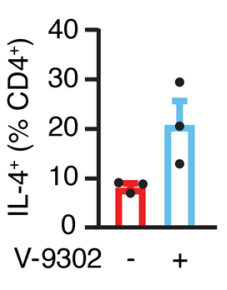

$\mathbf{K}$

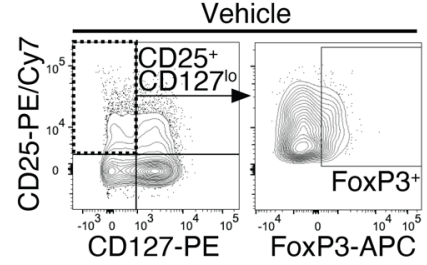

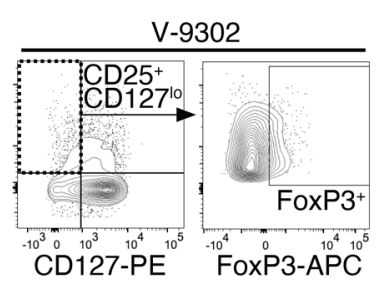

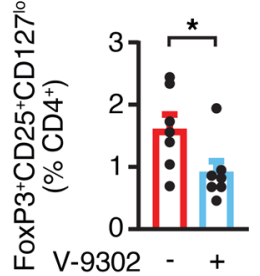

Figure 5. The glutamine transporter inhibitor V-9302 suppresses tumor growth and increases T lymphocyte activation in a model of TNBC. EO771 cells $\left(2.5 \times 10^{5}\right)$ were bilaterally injected into the number 4 mammary fat pads of female C57BL/6 mice (Taconic). Beginning on day 11 , mice were treated with vehicle (DMSO, red) or $50 \mathrm{mg} / \mathrm{kg} \mathrm{V-9302} \mathrm{(blue)} \mathrm{daily} \mathrm{for} 5$ days. (A) Tumor volume was measured over time. Arrow indicates beginning of treatment. $P<$ $1 \times 10^{-15}$ by 2-way ANOVA. $n=9-10$ mice per group. (B) Average tumor mass per mouse at harvest. $P=0.0060$ by unpaired Student's $t$ test. $n=9-10$ mice per group. (C) Immunofluorescence of tumor sections for Ki67 (top) or cleaved caspase-3 (bottom), both red. Nuclei were stained with DAPI (blue). Scale bars: $20 \mu \mathrm{m}$. Ki67+, cleaved caspase- $3^{+}$, and nuclei were averaged from 3 fields of view. Unpaired Student's $t$ test: $P=0.283$ (Ki67), $P=0.023$ (cleaved caspase-3). (D) Flow cytometric analyses of CD4+ (left) or CD8+ (right) T cells, plotted as a percentage of CD45+ immune cells, in vehicle- (red) or V-9302treated (blue) tumors. Unpaired Student's $t$ test: $P=0.023\left(C D 4^{+}\right), P=0.340$ (CD8 $\left.8^{+}\right) . n=5$ mice per group. (E) Immunohistochemistry of CD8a (brown) from vehicle- or V-9302-treated $(50 \mathrm{mg} / \mathrm{kg}$ ) tumors. Nuclei were stained with hematoxylin (blue). Edge (black) is considered $<500 \mu \mathrm{m}$ (denoted by solid line) from tumor margin, core (red) is $>500 \mu \mathrm{m}$. Scale bars: $500 \mu \mathrm{m}$ and $50 \mu \mathrm{m}$ for expanded and enlarged images, respectively. CD8 ${ }^{+}$cells (denoted by arrows) were averaged from 3 fields of view and normalized to field-of-view area. $P=0.019$ by unpaired Student's $t$ test. $n=3$ mice per group. (F-K) Flow

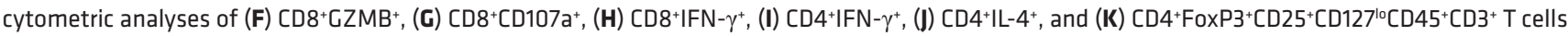
in vehicle- (red) or V-9302-treated (blue) tumors. Unpaired Student's $t$ test: $P=0.014$ (CD8+CZMB $), P=0.047\left(\right.$ CD8 ${ }^{+}$CD107a+), $P=0.007\left(\right.$ CD8 ${ }^{+}$IFN- $\left.\gamma^{+}\right), P=$ $0.0021\left(\mathrm{CD}^{+} \mathrm{IFN}-\gamma^{+}\right), P=0.057\left(\mathrm{CD}^{+} \mathrm{IL}-4^{+}\right), P=0.034\left(\mathrm{CD} 4^{+} \mathrm{FoxP3}^{+} \mathrm{CD} 25^{+} \mathrm{CD} 127^{10}\right) . n=3-8$ mice per group. ${ }^{*} P<0.05,{ }^{* *} P<0.01,{ }^{* * * *} P<0.001$. 
A

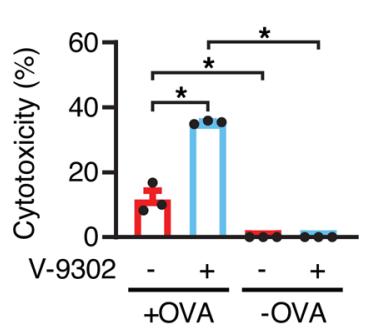

E

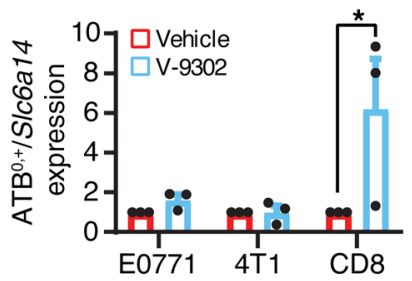

B

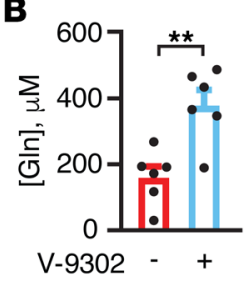

C

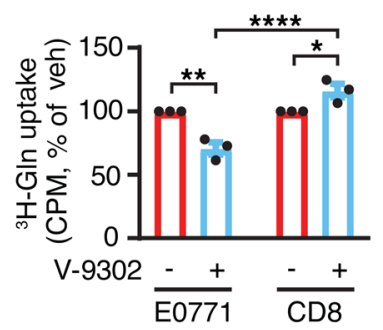

D

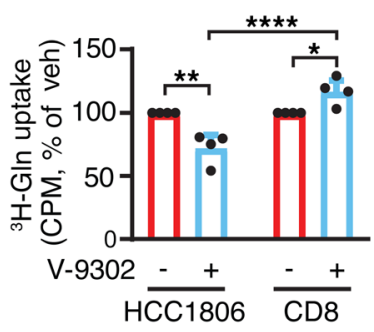

$\mathbf{F}$

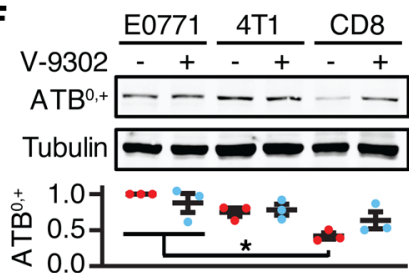

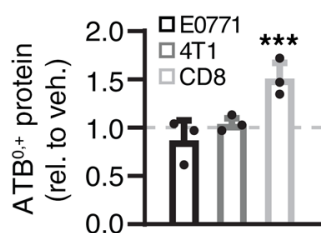

G

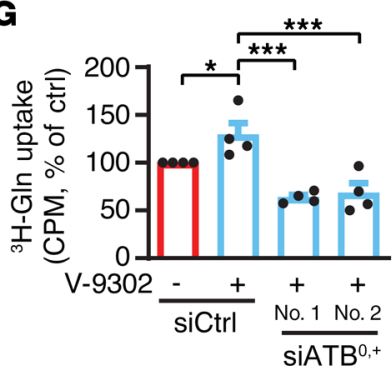

H

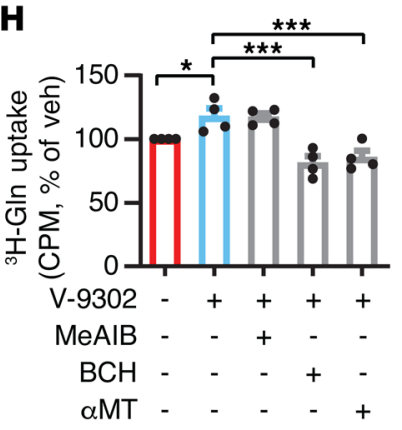

I

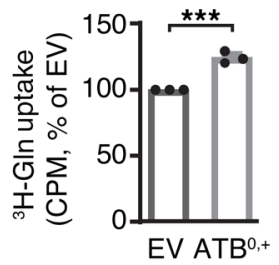

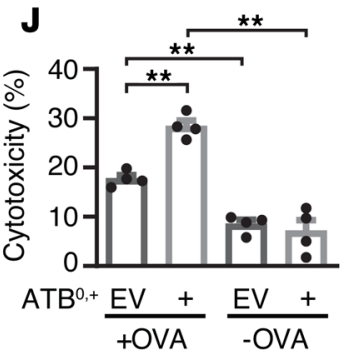

$\mathbf{K}$

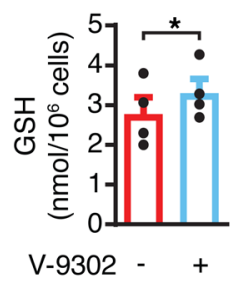

$\mathbf{L}$

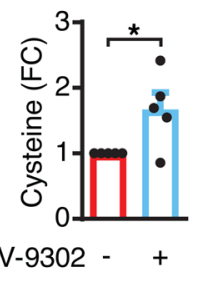

M

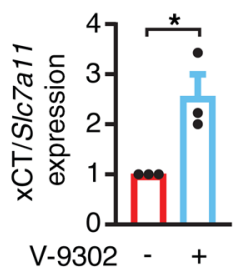

$\mathbf{N}$

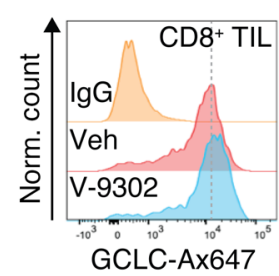

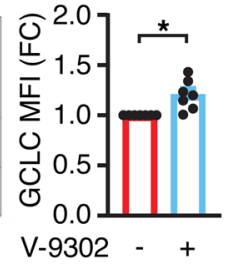

0

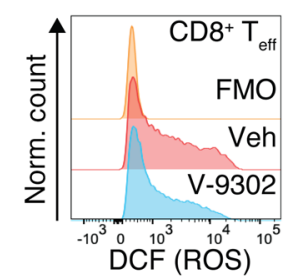

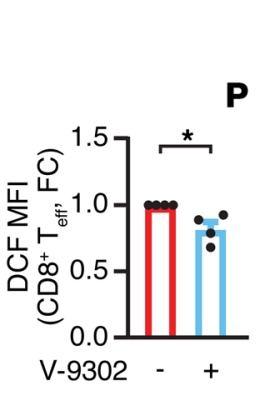

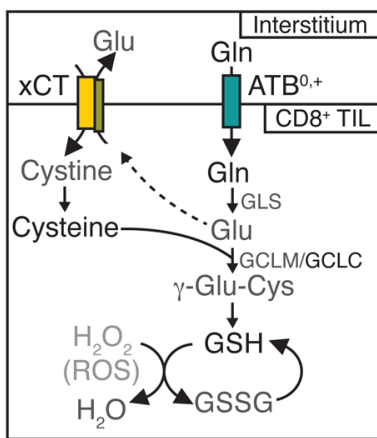

Figure 6. V-9302 induces ATB ${ }^{0,+}$ expression to sustain glutamine uptake and glutathione synthesis in activated CD8 ${ }^{+}$cells. (A) LDH cytotoxicity assay of E0771(OVA) and CD8 ${ }^{+}$OT-I CTLs cocultured in vehicle (red) or V-9302 (blue) $(n=3) . P=0.0018$ by 1-way ANOVA with Tukey's post hoc test. (B) Tumor interstitial fluid glutamine in vehicle- or V-9302-treated tumors. $P=0.0027$ by unpaired Student's $t$ test. $n=6$ mice per group. (C and $\mathbf{D}){ }^{3} \mathrm{H}$-glutamine uptake assay in (C) E0771 and C567BL/6 CD8 ${ }^{+}$CTLs or (D) HCC1806 and human CD8+ CTLs isolated from PBMCs in vehicle or V-9302 ( $\left.n=3-4\right)$. Averaged triplicate radioactivity (CPM) was normalized to vehicle. Two-way ANOVA (E0771: $P=2.18 \times 10^{-4}$; HCC1806: $P=1.43 \times 10^{-4}$ ) with Tukey's post hoc test. (E) Relative expression of ATB ${ }^{0,+} / S I c 6 a 14(n=3) . P=0.036$ by 2 -way ANOVA with Tukey's post hoc test. (F) ATB ${ }^{0,+}$ Western blot of cells from $\mathbf{E}^{\text {. Relative ATB }}$, $^{0,+}$ protein was normalized as indicated $(n=3)$. Two-way ANOVA (bottom, $P=0.0014$; right, $P=0.0026$ ) with Tukey's (bottom) or Sidak's (right) post hoc test. (G and H) ${ }^{3} \mathrm{H}$-glutamine uptake of $\mathrm{CD} 8^{+} \mathrm{CTLs}(\mathbf{C})$ after $A T B^{0,+}$ knockdown or $(\mathbf{H})$ in the presence of glutamine transporter inhibitors, as indicated $(n=4)$. One-way ANOVA: $P=4.98 \times 10^{-5}(\mathbf{C}), P=3.41 \times 10^{-4}(\mathbf{H})$; Holm-Sidak post hoc test. (I and J) $\mathrm{CD}^{+} \mathrm{CTL}^{-}$overexpressing ATB $\mathrm{B}^{0,+}$ were evaluated for $(\mathbf{I}){ }^{3} \mathrm{H}-\mathrm{glutamine}$ uptake or (J) LDH cytotoxicity $(n=3-4)$. Empty vector $(E V)$ was used as a control. Unpaired Student's $t$ test $\left(P=7.39 \times 10^{-4}\right)(I)$ or 1 -way ANOVA $(P=4.13 \times$ $10^{-5}$ ) with Tukey's post hoc test (J). (K and $\left.\mathbf{L}\right)$ Intracellular (K) glutathione (CSH) and (L) cysteine in CD8 ${ }^{+}$CTLs $(n=4-5)$. Paired $(P=0.020)(\mathbf{K})$ or unpaired $(P=0.027)(L)$ Student's $t$ test. $(M) \times C T / S / c 7 a 11$ expression in activated CD8 $8^{+}$CTLs $(n=3) . P=0.025$ by unpaired Student's $t$ test. (N) GCLC protein expression in CD8+ TILs in tumors of vehicle- or V-9302-treated mice. Dotted line denotes vehicle peak in representative data shown. $P=0.0034$ by unpaired Student's $t$ test. $n=7$ mice per group. (0) ROS in activated CD8 ${ }^{+} C D 44^{+}$CD62L- effector T cells determined by $\mathrm{H}_{2}$-DCFDA $(n=4)$. $P=0.016$ by unpaired Student's $t$ test. (P) Proposed model of V-9302-mediated increases (bold) and decreases (gray) in CD8 ${ }^{+}$TILs. ${ }^{*} P<0.05,{ }^{* *} P<0.01,{ }^{* * *} P<0.005,{ }^{* * * *} P<0.001$. 
tal Figure 7, A and B). Similar results were seen when this experiment was repeated in HCC1806 human TNBC cells cocultured with human peripheral $\mathrm{CD}^{+}$lymphocytes (Figure 6D), confirming that V-9302 affected glutamine uptake by TNBC cells, but not by CD ${ }^{+}$T cells. Importantly, expression of ASCT2 (Slc1a5), the sodium-coupled neutral amino acid transporter family members SNAT1 (Slc38a1) and SNAT2 (Slc38a2), as well as the L-type amino acid transporter LAT1 (Slc7a5) and its cotransporter 4F2HC (Slc3a2), were unchanged in E0771, 4T1, and CD8 ${ }^{+} \mathrm{T}$ cells in the presence of V-9302 (Supplemental Figure 7, C-G). Interestingly, the $\mathrm{Na}^{+} / \mathrm{Cl}^{-}$-dependent neutral and cationic amino acid transporter $\mathrm{ATB}^{0,+}$ (Slc6a14) was upregulated nearly 6-fold in $\mathrm{CD}^{+} \mathrm{T}$ cells, but not TNBC tumor cells, treated with V-9302 (Figure 6E). Despite low $\mathrm{ATB}^{0,+}$ protein expression in control $\mathrm{CD}^{+} \mathrm{T}$ cells, Western blot analysis confirmed $\mathrm{ATB}^{0,+}$ protein upregulation in $\mathrm{CD}^{+} \mathrm{T}$ cells in response to $\mathrm{V}-9302$ treatment to levels more comparable to those observed in TNBC cells (Figure 6F).

To assess whether $\mathrm{ATB}^{0,+} /$ Slc6a14 may compensate for glutamine transporter inhibition by $\mathrm{V}-9302$ in $\mathrm{CD}^{+} \mathrm{T}$ cells, we evaluated ${ }^{3} \mathrm{H}$-glutamine uptake in anti-CD3/anti-CD28-primed $\mathrm{CD}^{+}$ $\mathrm{T}$ cells following $\mathrm{ATB}^{0,+}$ knockdown (Figure 6G and Supplemental Figure $7 \mathrm{H})$. Consistent with our coculture system, V-9302 increases ${ }^{3} \mathrm{H}$-glutamine uptake in isolated $\mathrm{CD}^{+} \mathrm{T}$ cells (Figure 6G). However, ${ }^{3} \mathrm{H}$-glutamine uptake was significantly reduced following $\mathrm{ATB}^{\mathrm{O},+}$ knockdown (Figure 6G). Likewise, inhibition of $\mathrm{ATB}^{0,+}$ using 2-aminobicyclo(2.2.1)heptane-2-carboxylic acid (BCH) (57), an inhibitor of LAT1 and $\mathrm{ATB}^{0,+}$, or $\alpha$-methyl-DL-tryptophan $(\alpha \mathrm{MT})$, a selective $\mathrm{ATB}^{0,+}$ inhibitor (58), significantly reduced ${ }^{3} \mathrm{H}$-glutamine uptake in anti-CD3/anti-CD28-primed CD8 ${ }^{+} \mathrm{T}$ cells (Figure $6 \mathrm{H}$ ). In contrast, $\mathrm{N}$-methyl-aminoisobutyric acid (MeAIB), an inhibitor of SNAT1/ SNAT2 (49), had no effect on ${ }^{3} \mathrm{H}$-glutamine uptake (Figure $6 \mathrm{H}$ ), suggesting that $\mathrm{ATB}^{0,+} /$ Slc6a14 may be the primary transporter involved in glutamine influx of activated $\mathrm{CD}^{+} \mathrm{T}$ cells in the presence of V-9302. Overexpression of the the rat $\mathrm{ATB}^{0,+} /$ Slc 6 a14 ortho$\log$ in preactivated $\mathrm{CD} 8^{+} \mathrm{T}$ cells resulted in increased ${ }^{3} \mathrm{H}$-glutamine uptake at similar levels observed with V-9302 treatment (Figure 6I and Supplemental Figure 7, I and J) and conferred greater antigen-specific cytotoxicity to $\mathrm{CD}^{+} \mathrm{OT}-\mathrm{I}$ T cells against TNBC tumor cells in vitro (Figure 6J). Together, these results suggest that compensatory upregulation of the glutamine transporter $\mathrm{ATB}^{0,+} /$ Slc 6 a14 in V-9302-treated $\mathrm{CD}^{+} \mathrm{T}$ cells sustains glutamine uptake by $\mathrm{T}$ cells despite partial glutamine transporter inhibition, thus maintaining glutamine-dependent $\mathrm{T}$ cell cytotoxicity against tumor cells in V-9302-treated tumors. However, compensatory upregulation of glutamine transport was not seen in V-9302-treated TNBC cells, perhaps explaining their exquisite sensitivity to V-9302.

As a requirement to support effector functions, activated $\mathrm{CD}^{+} \mathrm{T}$ cells utilize glutamine to support numerous metabolic processes, including energy production and redox maintenance (21). To assess whether V-9302 impacts T cell energetics, we measured respiration of $\mathrm{CD}^{+}$TILs isolated from tumors treated with vehicle or V-9302 (Supplemental Figure 7, K and L). Although glutamine influx is enhanced in the presence of $\mathrm{V}-9302$, the drug does not change the oxygen consumption rate (OCR) of $\mathrm{CD}^{+}$TILs. However, ex vivo anti-CD3/anti-CD28-primed $C D 8^{+} \mathrm{T}$ cells cultured in $\mathrm{V}-9302$ are more dependent on glutamine to carry out respiration (Supplemental Figure 7M).
Because overall respiration was not affected, we next evaluated if V-9302 and enhanced glutamine influx improved the redox balance of $\mathrm{CD} 8^{+} \mathrm{T}$ cells by first evaluating glutathione levels. Indeed, anti-CD3/anti-CD28-primed CD8 ${ }^{+} \mathrm{T}$ cells cultured in V-9302 had increased glutathione levels compared with the vehicle control (Figure 6K). De novo glutathione synthesis involves ligation of glutamate with the amino acid cysteine by the glutamate-cysteine ligase (GCL). Consistent with increased glutathione production, activated $\mathrm{CD} 8^{+} \mathrm{T}$ cells cultured in $\mathrm{V}-9302$ showed increases in intracellular cysteine, but not alanine or asparagine, potentially through increased expression of the cysteine transporter, xCT (Figure 6, L and M, and Supplemental Figure 7, N and O). Expression of GCLC, the catalytic subunit of GCL, was also increased in the $\mathrm{CD}^{+}$and $\mathrm{CD} 4^{+}$TILs isolated from mice treated with V-9302 (Figure 6N and Supplemental Figure 7P). These increases in glutathione synthetic components following V-9302 treatment were coupled with a decrease in ROS levels of both $\mathrm{CD}^{+}$and $\mathrm{CD}^{+}$effector $\mathrm{T}$ cells (Figure $6 \mathrm{O}$ and Supplemental Figure 7Q). Together, these data suggest that V-9302 improves glutamine uptake to support de novo glutathione synthesis and improved redox balance in T cells (Figure 6P).

\section{Discussion}

Breast cancer is the most prevalent malignancy in women, encompassing $30 \%$ of all new cancer diagnoses, and the second leading cause of cancer-related death. Advances in targeted therapies have improved the survival of $\mathrm{ER}^{+}$and HER2 ${ }^{+}$breast cancers, but the prognosis of TNBC, the most aggressive and refractory subtype, remains grim. In search of vulnerabilities for these cancers, TNBCs were found to be dependent on glutamine metabolism (1519). However, key questions that are critical for drug development remain unanswered, including how tumor cell-derived glutamine metabolism effects immune function and whether inhibitors of GLS and glutamine transporters have an impact on antitumor immunity. In this report, we show that tumor cell-specific glutamine metabolism suppresses tumor-infiltrating $\mathrm{T}$ lymphocyte numbers and function, perhaps contributing to the nonresponsiveness of a significant number of TNBCs to immunotherapies observed in the clinic (59). Further, we show that TNBC tumors have increased susceptibility to V-9302, an ASCT2/SNAT2 inhibitor, whereas the CTLs upregulate alternative transporters to sustain glutamine uptake and effector function. Thus, preferential inhibition of glutamine metabolism in tumor cells may represent a promising targeted therapy that enhances antitumor immune responses in TNBC patients.

The tumor microenvironment is a diverse landscape, containing a variety of cell types including tumor cells, immune cell populations (lymphocytes, macrophages, natural killer cells, dendritic cells, myeloid-derived suppressor cells [MDSCs], etc.), fibroblasts, and endothelial cells among others $(60,61)$. Our data suggest that at least 2 of these populations, tumor cells and Tymphocytes, consume glutamine in the tumor interstitium. However, many of these additional populations also consume and utilize numerous metabolites, including glutamine. For example, M1 macrophage differentiation is favored upon glutamine synthetase inhibition due to greater accumulation of succinate (62), while GLS activity is crucial for endothelial cell proliferation and retinal 
angiogenesis in vivo (63). Therefore, the possibility remains that targeting tumor cell glutamine metabolism may alter additional cell populations present in the tumor microenvironment. Given that Rag1 deficiency essentially eliminated the antitumor effects of GLS loss of function, our results suggest that lymphocytes are the primary antitumor effectors in a glutamine-improved tumor microenvironment. However, other immune cells may also be affected by inhibition of glutamine metabolism. For example, the production of $\alpha$-ketoglutarate via glutaminolysis is important for activation of M2-like macrophages (64), and a prodrug of the glutamine antagonist DON was recently shown to inhibit generation and recruitment of MDSCs (65). Thus, although our findings of improved intratumoral $\mathrm{T}$ cell effector functions likely result from the increased glutamine availability to $\mathrm{T}$ cells, the potential remains for additional factors that can impact the immune system such as inhibition of suppressive microenvironments by M2-like macrophages and MDSCs.

The role of glutamine metabolism in T cells has been extensively studied in recent years, uncovering its requirement for processes such as proliferation, activation, and differentiation (21-23, $25,26)$. Glutamine uptake is massively enhanced upon TCR stimulation in $\mathrm{T}$ lymphocytes, and is crucial for their proliferation and cytokine production as glutamine starvation severely limits these downstream processes (22). Recent evidence suggests that preadaptation to glutamine deprivation in vitro enhances CD8 ${ }^{+}$ $\mathrm{T}$ cell responses when adoptively transferred in vivo $(26,66,67)$, although the underlying mechanisms were not completely understood. Such glutamine deprivation does not reflect conditions within the native tumor microenvironment, where there is a measurable levels of glutamine within the tumor interstitium. Using orthotopic and spontaneous mouse models, we show that loss of GLS in tumor cells increased interstitial glutamine concentrations to near physiological plasma levels $(41,43)$ and improved overall activation and effector capacity of $\mathrm{T}$ lymphocytes, indicating that reduced glutamine in the tumor microenvironment is immunosuppressive. Indeed, activity of effector $\mathrm{CD}^{+} \mathrm{T}$ cells measured by IFN- $\gamma$ secretion was increased in the presence of elevated glutamine. Likewise, pharmacological inhibition of glutamine transport using V-9302, which suppressed glutamine uptake in tumor cells but had little impact on $\mathrm{CD}^{+} \mathrm{T}$ cells, also improved $\mathrm{T}$ cell activation in vivo. Altogether, our results suggest that reducing glutamine metabolism specifically within tumor cells increases glutamine availability within the tumor microenvironment and improves $\mathrm{T}$ lymphocyte redox status and activation, supporting the hypothesis of glutamine competition within TNBC tumors.

Amino acid transporters involved in glutamine shuttling have been reported to have varying roles in $\mathrm{T}$ cell dynamics. In one report, loss of ASCT2 impaired both Th1 and Th17 differentiation, but enhanced Treg generation without a significant effect on $\mathrm{CD}^{+} \mathrm{T}$ cells (25). Using a different mouse line, a second report showed that ASCT2 deficiency did not affect either B cells or T cells (68), leaving open the question whether pharmacological inhibition of ASCT2 will affect lymphocyte function. While SNAT1/SLC38A1 and SNAT2/SLC38A2 expression is increased after TCR-mediated activation $(22,24,69)$, little is known regarding their roles in $\mathrm{T}$ cell development and function. Despite incomplete understanding of the mechanisms underlying phenotypes in the ASCT2/Slc1a5-deficient mouse models, the facts remain that (a) the glutamine transport inhibitor V-9302 was previously reported to have no impact on $\mathrm{CD} 44$ expression on $\mathrm{CD}^{+} \mathrm{T}$ cells in vitro (48), and (b) our in vivo data showed that administration of V-9302 in tumor-bearing mice increased $\mathrm{CD}^{+} \mathrm{T}$ cell activation and increased glutathione synthesis through upregulation of $\mathrm{ATB}^{0,+}$. A recent study showed that the glutamine antagonist DON also exhibits differential effects on tumor cells and T cells, but has broad impacts on a variety of metabolic pathways, including glutaminolysis and glycolysis (70), making it difficult to pinpoint the role of glutamine metabolism in these cell populations in vivo. Thus, the drug V-9302 or its analogs may represent an alternative approach to preferentially target tumor cells while sparing antitumor $\mathrm{T}$ cells.

Pharmacologically targeted therapies are capable of impacting multiple cell populations found within the tumor microenvironment as well as the human body. Due to the glutamine-addicted nature of TNBC cells, a great deal of attention has recently been focused on targeting glutamine metabolism as a means to therapeutically target these aggressive tumors. For example, the GLS inhibitor CB-839 is undergoing clinical trial testing for numerous solid tumors. Although CB-839 as a single-agent therapy has not produced promising results, the pleiotropic roles of glutamine suggest that CB-839 may be a good candidate for combination therapy to induce synthetic lethal effects on cancer cells. However, it may be that throttling the supply of glutamine would be needed in some settings. Our findings that V-9302 inhibits glutamine transport into tumor cells while enhancing $\mathrm{Th} 1$ and $\mathrm{CD} 8^{+} \mathrm{T}$ cell activation represents a potential therapeutic strategy to preferentially target glutamine metabolism in tumor cells. Our work suggests that strategies preferentially targeting glutamine metabolism in glutamine-addicted tumor cells may represent a more advantageous therapeutic approach to treating TNBC.

\section{Methods}

Flow cytometry. Immune cell populations were identified via flow cytometry from respective dissociated whole tumor cell suspensions or cultured cells. To assess mTORC1 signaling and ROS, C57BL/6 mouse (Jackson Laboratories) splenocytes were activated with anti-mouse CD3e (Tonbo, 70-0031; $1.25 \mu \mathrm{g} / \mathrm{mL}$ ) and anti-mouse CD28 (Tonbo, $70-0281 ; 1.25 \mu \mathrm{g} / \mathrm{mL}$ ) while treated with vehicle (DMSO) or V-9302 $(10 \mu \mathrm{M})$ for 48 hours. For phos-flow cytometry, cells were collected in the presence of phosphatase inhibitor and stained with 7-AAD (10 $\mu \mathrm{g} / \mathrm{mL}$ ). Fixed cells were permeabilized in cold methanol, and phospho-S6RP (Ser235/236; Cell Signaling Technology, 2211) was indirectly detected (Alexa Fluor 647-goat anti-rabbit secondary, 1:200, Invitrogen, A21244). T cells were identified by extracellular staining of mouse CD45, CD3e/TCR $\beta$, CD4, and CD8a (Supplemental Figure 3A and Supplemental Table 3). To detect ROS, cells were incubated with 5 $\mu \mathrm{M} \mathrm{H}_{2}$-DCFDA (Invitrogen, D399) at $37^{\circ} \mathrm{C}$ for 30 minutes. Dead cells were excluded using Ghost Dye Violet 510 (Tonbo, 13-0870).

For tumor immune analyses, tumors were harvested, weighed, and dissociated in RPMI-1640, 5\% FBS, collagenase IA $(1 \mathrm{mg} / \mathrm{mL}$; Sigma-Aldrich, C9891), and DNase I (0.25 mg/mL; Sigma-Aldrich, DN25) for 30 minutes at $37^{\circ} \mathrm{C}$. To obtain single-cell suspensions, digested tissue was filtered through a $70 \mu \mathrm{m}$ cell strainer and red blood cells were lysed using ACK lysis buffer. For detection of IFN- $\gamma, 1 \times 10^{6}$ to 
$2 \times 10^{6}$ cells were stimulated in RPMI-1640 containing 5\% FBS, phorbol 12-myristate 13-acetate (PMA) (50 ng/mL; Sigma-Aldrich, P8139), ionomycin $(1 \mu \mathrm{g} / \mathrm{mL}$; Sigma-Aldrich, I0634), and GolgiPlug protein transport inhibitor (1:1000; BD, 555029) for 4 hours at $37^{\circ} \mathrm{C}$. Dead cells were excluded from analysis by staining with Ghost Dye Violet 450 (Tonbo, 13-0863) or Violet 510, as indicated. Following blocking with anti-CD16/32 (mouse Fc block), extracellular staining was achieved against the following proteins: CD45, CD45.2, CD3e, TCR $\beta$, CD4, CD8a, CD25, CD69, CD44, CD62L, CD127, and/or CD107a (Supplemental Table 3). Intracellular staining for GZMB, IFN- $\gamma$, IL-4, IL-17A, and GCLC was accomplished using a Cytofix/Cytoperm solution kit (BD, 554714) on paraformaldehyde-fixed cells, per the manufacturer's directions. GCLC was indirectly detected by Alexa Fluor 647-goat anti-rabbit secondary antibody as described above. To detect FoxP3, fixed cells were stained using the FoxP3/Transcription Factor Staining Kit (Tonbo, TNB-0607), as directed. Isolated splenocytes or tumor cell suspensions were used for unstained, compensation, fluorescence minus one (FMO), and isotype controls, where appropriate. Flow cytometry data were obtained on a BD Fortessa using BD FACSDiva software and analyzed using FlowJo software.

To assess T cell infiltration, E0771(OVA) sgLacZ (WT) or sgGls3 $\left(\mathrm{GLS}^{\mathrm{KO}}\right)\left(5 \times 10^{5}\right.$ cells) were bilaterally implanted into the number 4 mammary fat pads of 6- to 8-week-old C57BL/6 female mice (Jackson Laboratories or Taconic as indicated in the figure legends). OT-I splenocytes were isolated from 4-week-old female OT-I mice (Jackson Laboratories) and activated with SIINFEKL peptide $(1 \mu \mathrm{g} / \mathrm{mL}$; Invivogen) for 72 hours. Following activation, CD $8^{+}$OT-I T cells were isolated using mouse CD8a microbeads (Miltenyi Biotec, 130-045201) as described by the manufacturer and then transduced with $\mathrm{MiG}$ retrovirus to overexpress GFP. GFP ${ }^{+} \mathrm{CD}^{+} \mathrm{OT}$-I T cells $\left(3 \times 10^{6}\right)$ were adoptively transferred via retro-orbital injection into tumor-bearing mice 8 days following tumor implantation. After 3 days, tumors were harvested, processed, and analyzed by flow cytometry as described above. Dead cells were excluded from analysis by staining with Ghost Dye Violet 510. CD8 ${ }^{+}$OT-I cells were detected by GFP.

Tumor interstitial fluid metabolic assays. Tumor interstitial fluid was collected from tumors as previously described (71), with some changes. Briefly, tumors were harvested, weighed, and then washed with $1 \times$ PBS to remove excess blood from the tumor surface. Excess PBS was removed by carefully blotting with a Kimwipe. Tumors were placed in a $20 \mu \mathrm{m}$ pluriStrainer (pluriSelect) mounted in a microcentrifuge tube, then centrifuged at $100 \mathrm{~g}$ at $4^{\circ} \mathrm{C}$ for 30 minutes. The cleared interstitium was deproteinated and glutamine was detected using the Glutamine Detection Assay Kit (Abcam, ab197011) according to the manufacturer's instructions. Known glutamine standards were used to calculate concentrations.

Intracellular amino acid assays. To assess intracellular cysteine, alanine, and asparagine levels, anti-CD3/anti-CD28-activated CD8 ${ }^{+}$ $\mathrm{T}$ cells freshly isolated from C57BL/6 mice were cultured in vehicle or V-9302, as described above. Equal numbers of cells were homogenized and analyzed for cysteine (Abcam, ab211099), alanine (SigmaAldrich, MAK001), or asparagine (BioVision, K736) as described by the manufacturers. Tumor cell intracellular glutamate concentrations were determined using the Glutamate Assay Kit (Sigma-Aldrich, MAK004-1KT) as previously described (6) with some changes. Briefly, control or GLS loss-of-function E0771 single-cell clones were cultured in glutamine-free, serum-free DMEM for 24 hours. Cells were then stimulated with L-glutamine $(2.5 \mathrm{mM})$ for 15 minutes. Fluorescence or absorbance was measured using the BioTek Synergy HT plate reader. Amino acid concentrations were determined using known standards and are reported as fold change using the appropriate control.

IFN- $\gamma$ cytokine secretion by Tc1 cells in response to glutamine. $\mathrm{CD} 8^{+}$ $\mathrm{T}$ cells were purified from spleens of C57BL/6 mice and activated with plate-bound anti-CD3 $(2.5 \mu \mathrm{g} / \mathrm{mL})$ and soluble anti-CD28 (2.5 $\mu \mathrm{g} / \mathrm{mL}$ ) in the presence of irradiated antigen-presenting cells (APCs). $\mathrm{CD}^{+} \mathrm{T}$ cells were cultured at $1 \times 10^{6}$ cells per $\mathrm{mL}$ in $2 \mathrm{~mL}$ on 12 -well plates with $2 \times 10^{6}$ irradiated APCs and fed rh-IL2 $(20 \mathrm{ng} / \mathrm{mL})$ every other day. Cells were split on day 2 into 6-well plates and cultured for a total of 4 days. On day 4 , cultures were harvested, counted, and equal numbers of cells were replated at a density of $2 \times 10^{6}$ cells $/ \mathrm{mL}$ in media with indicated concentrations of glutamine, reactivated with fresh anti-CD3 and anti-CD28 $(1.25 \mu \mathrm{g} / \mathrm{mL}$ each), and cultured overnight in a $37^{\circ} \mathrm{C} \mathrm{CO}_{2}$ incubator. Culture supernatants were collected after 18-24 hours of culture for anti-IFN- $\gamma$ cytokine sandwich ELISA, as previously described (72), using anti-mouse IFN- $\gamma$ (BD, 551216) and anti-mouse IFN- $\gamma$-biotin (BD, 554410) as capture and detection antibodies, respectively.

Cytotoxicity assay. For in vitro cytotoxicity assays, E0771(OVA) or E0771 $\left(1 \times 10^{4}\right.$ per well) were plated in triplicate as indicated and allowed to attach for 6 hours. Tumor cells were cocultured with SIINFEKL-activated WT or MiG-ATB ${ }^{0,+}-$ transduced CD8 ${ }^{+}$OT-I cells (2 $\times 10^{3}$ per well, resulting in 5:1 tumor cell to $\mathrm{CD}^{+}$cell ratio) in $\mathrm{T}$ cell media in the absence or presence of V-9302 $(10 \mu \mathrm{g} / \mathrm{mL})$, as indicated, for 48 hours. Cytotoxicity was measured using the LDH Cell-mediated Cytotoxicity Assay (Thermo Fisher Scientific), according to the manufacturer's directions. Percentage cytotoxicity was calculated following correction for spontaneous LDH release from tumor cells and CD8 ${ }^{+}$ $\mathrm{T}$ cells alone and based on maximal LDH release for each condition.

Glutamine uptake assay. C57BL/6 or OT-I mouse $\mathrm{CD}^{+}$cells were isolated, activated, and modified, as indicated. Human PMBCs were activated with functional grade anti-human CD3 $(1 \mu \mathrm{g} / \mathrm{mL}$; Invitrogen, 16-0038) and anti-human CD28 $(1 \mu \mathrm{g} / \mathrm{mL}$; Invitrogen, 16-0289), with supplementation of human IL-2 (20 U/mL) for 48 hours and extracted using human CD8 microbeads (Miltenyi Biotec, 130-045-201) as directed. E0771 $\left(3.5 \times 10^{4}\right.$ per well) or HCC1806 $\left(1.0 \times 10^{4}\right.$ per well $)$ cells were plated in at least duplicate in a 96-well plate, and cells were allowed to attach overnight, as indicated. Glutamine uptake was measured in tumor or $\mathrm{CD}^{+}$cells in isolation or tumor/CD8 ${ }^{+}$cell cocultures (1:1) as described previously (48), with a few alterations including omission of $\mathrm{BCH}$ except where specified. Briefly, tumor cells and/or CD8 ${ }^{+}$cells were washed 3 times in uptake buffer (10 mM HEPES pH 7.4, $137 \mathrm{mM} \mathrm{NaCl}, 5.1 \mathrm{mM} \mathrm{KCl,} 0.77 \mathrm{mM}$ $\mathrm{KH}_{2} \mathrm{PO}_{4}, 0.71 \mathrm{mM} \mathrm{MgSO}_{4}, 1.1 \mathrm{CaCl}_{2}$, and $10 \mathrm{mM}$ D-glucose). CD8 ${ }^{+} \mathrm{T}$ cells were resuspended in assay buffer containing V-9302 $(10 \mu \mathrm{M})$, MeAIB (10 mM) (Sigma-Aldrich, M2383), BCH (500 $\mu \mathrm{M})$ (Cayman Chemical, 15249), and/or $\alpha$ MT (100 $\mu$ M) (Sigma-Aldrich, M8377), as indicated, along with the appropriate vehicles. For coculture assays, $\mathrm{CD}^{+}$cells were directly added to tumor cells. Following a 15-minute incubation at $37^{\circ} \mathrm{C}, 1 \mu \mathrm{Ci}$ of L-[2,3,4-3H]-glutamine (American Radiolabeled Chemicals, ART-0149) was added, and cells were incubated an additional 15 minutes. Suspended $\mathrm{CD}^{+}$cells were removed, and both tumor and $\mathrm{T}$ cells were washed in uptake buffer separately. Cells were lysed with $1 \mathrm{~N} \mathrm{NaOH}$, and radioactivity was measured using a Beckman LS 6500 Scintillation counter for 1 min- 
ute and recorded as counts per minute (CPM). Treated samples were normalized to the appropriate controls.

Glutathione assay. $\mathrm{CD}^{+} \mathrm{T}$ cells were activated and cultured in the presence of vehicle or V-9302, as described above. Glutathione was measured using the GSH-Glo Glutathione Assay (Promega, V6911) according to the manufacturer's instructions. Luminescence was measured using the BioTek Synergy HT plate reader. Glutathione concentrations were determined from a known standard.

Quantitative real-time polymerase chain reaction. E0771 or $4 \mathrm{~T} 1$ cells $\left(5 \times 10^{5}\right)$ were plated and treated with vehicle or $10 \mu \mathrm{M} \mathrm{V}-9302$ for 48 hours. Likewise, isolated activated primary mouse splenocytes were treated with vehicle or $\mathrm{V}-9302$, followed by isolation of $\mathrm{CD} 8^{+}$cells as described above. RNA was collected using the RNeasy kit (Qiagen), and cDNA was generated using the iScript cDNA synthesis kit (BioRad) according to the manufacturers' directions. Samples were amplified in triplicate using SYBR Green PCR Master Mix (Thermo Fisher Scientific), $10 \mathrm{pmol}$ of primer (Supplemental Table 4), and $20 \mathrm{ng}$ of cDNA per reaction with the StepOnePlus (Applied Biosystems). Quantitation was performed using the $\Delta \Delta \mathrm{C}_{\mathrm{t}}$ method.

Gene expression and survival data set analysis. TCGA Breast Adenocarcinoma (BRCA) database (HiSeqV2), downloaded January 19, 2018 from the UCSC Xena platform (73) (https://xena.ucsc.edu), was searched for mRNA expression and $z$-scores were calculated based on all tumor samples. The average $z$-scores for glutaminase $(G L S)$, asparagine synthetase (ASNS), glutamine-fructose-6-phosphate transaminase 1 (GFPT1), ASCT2 (SLC1A5), SNAT1 (SLC38A1), SNAT2 (SLC38A2), and $\mathrm{ATB}^{0,+}$ (SLC6A14) comprise the glutamine metabolism gene signature (GMGS). The average $z$-score of the glycolysis genes ALDOA, ENO1, GAPDH, GPI, HK1, LDHA, PFKM, PFKL, PGAM1, PKM2, and TPI1 as well as the GLUT1 transporter (SLC2A1) generated the glycolysis gene signature. To avoid confounding data from highly glycolytic tumors, the BLBC samples (PAM50, ref. 74) with expression profiles signifying the greatest dependence on glutamine metabolism ("glutamine metabolism high") were identified based on glutamine metabolism and glycolysis gene signatures (top 12.5\%). All other BLBC tumors were classified as having "mixed metabolism." A CTL gene signature was calculated by averaging the $z$-scores of GZMA, GZMB, PRF1, and IFNG expression.

For survival analyses, the glutamine metabolism gene signature (GLS, 211414_at; ASNS, 205047_s_at; GFPT1, 202722_s_at; SLC1A5, 208916_at; SLC38A1, 218237_s_at; SLC38A2, 220924_s_at; SLC6A14, 219795_at) and the CTL gene signature (GZMA, 205488_at; GZMB, 210164_at; PRF1, 214617_at; IFNG 210354_at) gene expression and overall survival (OS) and distant metastasis-free survival (DMFS) data from basal breast cancer tumors were downloaded from KM plot (75) (http://www.kmplot.com). Automatic cutoff was used to define "high" versus "low" expression, with cutoff values defined as followed: GLS (OS: 57, range $=2-587$ ) (DMFS: 43, range $=2-242$ ), ASNS (OS: 1560, range $=109-13,370)($ DMFS: 902, range $=33-13,370)$, GFPT1 (OS: 1726, range $=354-5352)$ (DMFS: 1138, range $=377-5352)$, SLC1A5 (OS: 380, range $=131-2039)($ DMFS: 709 , range $=131-2039)$, SLC38A1 (OS: 3704, range $=94-16,075)($ DMFS: 2817, range $=370-14,915)$, SLC38A2 (OS: 2469 , range $=288-13,482)$ (DMFS: 2976, range $=751-7711$ ), SLC6A14 (OS: 899, range $=1-8829)$ (DMFS: 920, range $=3-10,359$ ), GZMA (OS: 239, range $=17-4627$ ) (DMFS: 272, range $=46-4627$ ), GZMB (OS: 289, range $=7-6030)$ (DMFS: 295, range $=18-6030$ ), PRF1 (OS: 201, range $=46-6453)($ DMFS: 203 , range $=56-6453)$, and
IFNG (OS: 58, range $=1-668$ ) (DMFS: 68, range $=2-665)$. Characterization of "high" expression of the CTL gene signature required "high expression" classification of at least 2 genes, while characterization of "high" expression of the glutamine metabolism gene signature required "high expression" classification of at least 4 genes. Samples were then categorized based on the glutamine metabolism gene signature or GLS expression and the CTL gene signature.

Statistics. For survival curve analysis, the Mantel-Cox method was used for log-rank analysis of all groups, with the Bonferroni correction utilized for multiple comparisons where applicable. Hazard ratios (HRs) and 95\% confidence intervals were calculated for single comparisons using the log-rank test.

For dose-response analyses, nonlinear fit was applied to both CB-839- and V-9302-treated cells. To directly assess differences between CB-839 and V-9302, unpaired Student's $t$ test was applied at the indicated points.

All plots and statistical analyses were completed using GraphPad Prism software. Data from independent assays (dot) as well as summary data (mean with SEM) are shown. For tumor experiments, data are reported from 1-2 independent experiments, with each dot representing a single animal. For comparisons between 2 groups, unpaired or paired Student's $t$ tests were performed as indicated. For multiple comparisons, 1- or 2-way analysis of variance (ANOVA) was performed with post hoc analysis using Tukey's, Dunnett's, HolmSidak's, or Sidak's multiple-comparison tests, as indicated. Outliers were identified using the ROUT method $(Q=5 \%)$. All statistical tests were completed as 2-tailed, and $P$ less than 0.05 was considered statistically significant, except where corrected for multiple comparisons as indicated. All error bars are SEM.

Study approval. All mouse procedures were performed under protocols approved by Vanderbilt University's Institutional Animal Care and Utilization Committee (IACUC).

\section{Author contributions}

DNE and JC conceptualized the project. DNE, VMN, ALR, SW, $\mathrm{YH}, \mathrm{LCK}$, and YP performed the experiments and analyzed the data. SHC, QW, SZ, and MRB provided technical and conceptual support. JCR, HCM, and RSC provided critical reagents. DNE, ALR, RSC, MRB, and JC wrote the manuscript.

\section{Acknowledgments}

We thank Barbara Fingleton (Vanderbilt University) for providing E0771 cells and David DeNardo (Washington University) for providing the pLX-Hygro-IRES-OVA vector. Flow cytometry experiments were performed in the VUMC Flow Cytometry Shared Resource, which is supported by the Vanderbilt Ingram Cancer Center (P30 CA68485) and the Vanderbilt Digestive Disease Research Center (DK058404). This work was supported by NIH grants R01 CA177681 (to JC), R01 CA95004 (to JC), R01 CA250506 (to JC), T32 CA009592 (to DNE); a VA Merit Award 5101BX000134 and a VA Senior Research Career Scientist Award IK6BX005391 (to JC); and a Susan G. Komen Postdoctoral Fellowship Award PDF 17480733 (to DNE).

Address correspondence to: Jin Chen, T-3207E, MCN, Vanderbilt University Medical Center, Nashville, Tennessee 37232, USA. Phone: 615.343.3819; Email: jin.chen@vanderbilt.edu. 
1. Wise DR, Thompson CB. Glutamine addiction: a new therapeutic target in cancer. Trends Biochem Sci. 2010;35(8):427-433.

2. Hensley CT, et al. Glutamine and cancer: cell biology, physiology, and clinical opportunities. J Clin Invest. 2013;123(9):3678-3684.

3. O'Sullivan D, Pearce EL. Targeting T cell metabolism for therapy. Trends Immunol. 2015;36(2):71-80.

4. Palmer CS, et al. Glucose metabolism regulates $\mathrm{T}$ cell activation, differentiation, and functions. Front Immunol. 2015;6:1.

5. Ramsay G, Cantrell D. Environmental and metabolic sensors that control T cell biology. Front Immunol. 2015;6:99.

6. Edwards DN, et al. The receptor tyrosine kinase EphA2 promotes glutamine metabolism in tumors by activating the transcriptional coactivators YAP and TAZ. Sci Signal. 2017;10(508):eaan4667.

7. Youngblood VM, et al. The Ephrin-A1/EPHA2 signaling axis regulates glutamine metabolism in HER2-positive breast cancer. Cancer Res. 2016;76(7):1825-1836.

8. Ngwa VM, et al. Microenvironmental metabolism regulates antitumor immunity. Cancer Res. 2019;79(16):4003-4008.

9. Nakazawa MS, et al. Oxygen availability and metabolic adaptations. Nat Rev Cancer. 2016;16(10):663-673.

10. Hanahan D, Weinberg RA. Hallmarks of cancer: the next generation. Cell. 2011;144(5):646-674.

11. Luo M, et al. Asparagine and glutamine: co-conspirators fueling metastasis. Cell Metab . 2018;27(5):947-949.

12. Zhang J, et al. Cancer cell metabolism: the essential role of the nonessential amino acid, glutamine. EMBO J. 2017;36(10):1302-1315.

13. Platten $\mathrm{M}$, et al. Tryptophan metabolism as a common therapeutic target in cancer, neurodegeneration and beyond. Nat Rev Drug Discov. 2019;18(5):379-401.

14. Lukey MJ, et al. Targeting amino acid metabolism for cancer therapy. Drug Discov Today. 2017;22(5):796-804.

15. Winnike JH, et al. Stable isotope-resolved metabolomic differences between hormone-responsive and triple-negative breast cancer cell lines. Int $J$ Breast Cancer. 2018;2018:2063540.

16. Altman BJ, et al. From Krebs to clinic: Glutamine metabolism to cancer therapy. Nat Rev Cancer. 2016;16(10):619-634.

17. Lampa $\mathrm{M}$, et al. Glutaminase is essential for the growth of triple-negative breast cancer cells with a deregulated glutamine metabolism pathway and its suppression synergizes with mTOR inhibition. PLoS One. 2017;12(9):e0185092.

18. Kim S, et al. Expression of glutamine metabolism-related proteins according to molecular subtype of breast cancer. Endocr Relat Cancer. 2013;20(3):339-348.

19. Van Geldermalsen M, et al. ASCT2/SLC1A5 controls glutamine uptake and tumour growth in triple-negative basal-like breast cancer. Oncogene. 2016;35(24):3201-3208.

20. Wang J-B, et al. Targeting mitochondrial glutaminase activity inhibits oncogenic transformation. Cancer Cell. 2010;18(3):207-219.
21. Buck MD, et al. $\mathrm{T}$ cell metabolism drives immunity. J Exp Med. 2015;212(9):1345-1360.

22. Carr EL, et al. Glutamine uptake and metabolism Are coordinately regulated by ERK/MAPK during T lymphocyte activation. J Immunol. 2010;185(2):1037-1044.

23. Sinclair LV, et al. Control of amino-acid transport by antigen receptors coordinates the metabolic reprogramming essential for $\mathrm{T}$ cell differentiation. Nat Immunol. 2013;14(5):500-508.

24. Ron-Harel N, et al. T cell activation depends on extracellular alanine. Cell Rep. 2019;28(12):3011-3021.e4.

25. Nakaya $\mathrm{M}$, et al. Inflammatory $\mathrm{T}$ cell responses rely on amino acid transporter ASCT2 facilitation of glutamine uptake and mTORC1 kinase activation. Immunity. 2014;40(5):692-705.

26. Johnson MO, et al. Distinct regulation of Th17 and Th1 cell differentiation by glutaminasedependent metabolism. Cell. 2018;175(7):1780-1795.e19.

27. Klysz D, et al. Glutamine-dependent $\alpha$-ketoglutarate production regulates the balance between $\mathrm{T}$ helper 1 cell and regulatory $\mathrm{T}$ cell generation. $\mathrm{Sci}$ Signal. 2015;8(396):ra97.

28. Siska PJ, Rathmell JC. T cell metabolic fitness in antitumor immunity. Trends Immunol. 2015;36(4):257-264.

29. Cluntun AA, et al. Glutamine metabolism in cancer: understanding the heterogeneity. Trends Cancer. 2017;3(3):169-180.

30. O'Sullivan D, et al. Metabolic interventions in the immune response to cancer. Nat Rev Immunol. 2019;19(5):324-335.

31. Chang $\mathrm{C}-\mathrm{H}$, et al. Metabolic competition in the tumor microenvironment is a driver of cancer progression. Cell. 2015;162(6):1229-1241.

32. Ho P-C, et al. Phosphoenolpyruvate is a metabolic checkpoint of anti-tumor $\mathrm{T}$ cell responses. Cell. 2015;162(6):1217-1228.

33. Bertucci F, et al. How basal are triple-negative breast cancers? Int J Cancer. 2008;123(1):236-240.

34. Johnstone $\mathrm{CN}$, et al. Functional and molecular characterisation of EO771.LMB tumours, a new C57BL/6-mouse-derived model of spontaneously metastatic mammary cancer. Dis Model Mech. 2015;8(3):237-251.

35. Mombaerts P, et al. RAG-1-deficient mice have no mature B and T lymphocytes. Cell. 1992;68(5):869-877.

36. Green JE, et al. The C3(1)/SV40 T-antigen transgenic mouse model of mammary cancer: ductal epithelial cell targeting with multistage progression to carcinoma. Oncogene. 2000;19(8):1020-1027.

37. Herschkowitz JI, et al. Identification of conserved gene expression features between murine mammary carcinoma models and human breast tumors. Genome Biol. 2007;8(5):R76.

38. Deeb KK, et al. Identification of an integrated SV40 T/t-antigen cancer signature in aggressive human breast, prostate, and lung carcinomas with poor prognosis. Cancer Res. 2007;67(17):8065-8080.

39. Wagner KU, et al. Cre-mediated gene deletion in the mammary gland. Nucleic Acids Res. 1997;25(21):4323-4330.

40. Heusel JW, et al. Cytotoxic lymphocytes require granzyme $\mathrm{B}$ for the rapid induction of DNA fragmentation and apoptosis in allogeneic target cells. Cell. 1994;76(6):977-987.

41. Bagga P, et al. Characterization of cerebral glutamine uptake from blood in the mouse brain: Implications for metabolic modeling of 13C NMR data. J Cereb Blood Flow Metab. 2014;34(10):1666-1672.

42. Maggs DG, et al. Interstitial fluid concentrations of glycerol, glucose, and amino acids in human quadricep muscle and adipose tissue: evidence for significant lipolysis in skeletal muscle. JClin Invest. 1995;96(1):370-377.

43. Kew S, et al. Dietary glutamine enhances murine T-lymphocyte responsiveness. J Nutr. 1999;129(8):1524-1531.

44. Rötzschke $\mathrm{O}$, et al. Exact prediction of a natural $\mathrm{T}$ cell epitope. Eur JImmunol. 1991;21(11):2891-2894.

45. Hogquist KA, et al. T cell receptor antagonist peptides induce positive selection. Cell. 1994;76(1):17-27.

46. Carbone FR, et al. T cell receptor $\alpha$-chain pairing determines the specificity of residue 262 within the kb-restricted, ovalbumin257-264 determinant. Int Immunol. 1992;4(8):861-867.

47. Parlati F. Anti-tumor acitivity of glutaminase inhibitor CB-839 in combination with clinical anti-cancer agents. Presented at: Keystone Symposium on Tumor Metababolism; January 21-25, 2018; Snowbird, Utah, USA. https://www. calithera.com/wp-content/uploads/2018/02/ Keystone-Metabolism-2018-parlati-final.pdf. Accessed December 16, 2020.

48. Schulte ML, et al. Pharmacological blockade of ASCT2-dependent glutamine transport leads to antitumor efficacy in preclinical models. Nat Med. 2018;24(2):194-202.

49. Bröer A, et al. Disruption of amino acid homeostasis by novel ASCT2 inhibitors involves multiple targets. Front Pharmacol. 2018;9:785

50. Ayers M, et al. IFN- $\gamma$-related mRNA profile predicts clinical response to PD-1 blockade. JClin Invest. 2017;127(8):2930-2940.

51. Lohneis $P$, et al. Cytotoxic tumour-infiltrating T lymphocytes influence outcome in resected pancreatic ductal adenocarcinoma. Eur J Cancer. 2017;83:290-301.

52. Peranzoni E, et al. Macrophages impede CD8 T cells from reaching tumor cells and limit the efficacy of anti-PD-1 treatment. Proc Natl Acad Sci U S A. 2018;115(17):E4041-E4050.

53. Klebanoff CA, et al. Central memory self/ tumor-reactive $\mathrm{CD} 8^{+} \mathrm{T}$ cells confer superior antitumor immunity compared with effector memory T cells. Proc Natl Acad Sci U S A. 2005;102(27):9571-9576.

54. Klebanoff CA, et al. IL-15 enhances the in vivo antitumor activity of tumor-reactive CD8 ${ }^{+}$T Cells. Proc Natl Acad Sci U S A. 2004;101(7):1969-1974.

55. Delgoffe GM, et al. The mTOR kinase differentially regulates effector and regulatory $\mathrm{T}$ cell lineage commitment. Immunity. 2009;30(6):832-844.

56. Powell JD, et al. Inhibition of cell cycle progression by rapamycin induces $\mathrm{T}$ cell clonal anergy even in the presence of costimulation. J Immunol. 1999;162(5):2775-2784.

57. Kim CS, et al. BCH, an inhibitor of system L amino acid transporters, induces apoptosis in cancer 
cells. Biol Pharm Bull. 2008;31(6):1096-1100.

58. Sloan JL, Mager S. Cloning and functional expression of a human $\mathrm{Na}+$ and $\mathrm{Cl}(-)$-dependent neutral and cationic amino acid transporter $\mathrm{BO}+$. J Biol Chem. 1999;274(34):23740-23745.

59. Marra A, et al. Recent advances in triple negative breast cancer: the immunotherapy era. $B M C$ Med. 2019;17(1):90.

60. Wang M, et al. Role of tumor microenvironment in tumorigenesis. J Cancer. 2017;8(5):761-773.

61. Balkwill FR, et al. The tumor microenvironment at a glance. J Cell Sci. 2012;125(23):5591-5596.

62. Palmieri EM, et al. Pharmacologic or genetic targeting of glutamine synthetase skews macrophages toward an M1-like phenotype and inhibits tumor metastasis. Cell Rep. 2017;20(7):1654-1666.

63. Rohlenova K, et al. Endothelial cell metabolism in health and disease. Trends Cell Biol. 2018;28(3):224-236.

64. Liu PS, et al. $\alpha$-ketoglutarate orchestrates macrophage activation through metabolic and epigenetic reprogramming. Nat Immunol. 2017;18(9):985-994.

65. Oh MH, et al. Targeting glutamine metabolism enhances tumor-specific immunity by modulating suppressive myeloid cells. JClin Invest. 2020;130(7):3865-3884.

66. Suzuki J, et al. The tumor suppressor menin prevents effector CD8 T-cell dysfunction by targeting mTORC1-dependent metabolic activation. Nat Commun. 2018;9(1):3296.

67. Nabe S, et al. Reinforce the antitumor activity of $\mathrm{CD}^{+} \mathrm{T}$ cells via glutamine restriction. Cancer Sci. 2018;109(12):3737-3750.

68. Masle-Farquhar E, et al. ASCT2 (SLC1A5)deficient mice have normal B-cell development, proliferation, and antibody production. Front Immunol. 2017;8:549.

69. Matheson NJ, et al. Cell surface proteomic map of HIV infection reveals antagonism of amino acid metabolism by Vpu and Nef. Cell Host Microbe. 2015;18(4):409-423.
70. Leone RD, et al. Glutamine blockade induces divergent metabolic programs to overcome tumor immune evasion. Science. 2019;366(6468):1013-1021.

71. Wiig $\mathrm{H}$, et al. Isolation of interstitial fluid from rat mammary tumors by a centrifugation method. Am J Physiol Heart Circ Physiol. 2003;284(1):H416-H424.

72. Kemp RA, Ronchese F. Tumor-specific Tc1, but not Tc2, cells deliver protective antitumor immunity. J Immunol. 2001;167(11):6497-6502.

73. Goldman MJ, et al. Visualizing and interpreting cancer genomics data via the Xena platform. Nat Biotechnol. 2020;38(6):675-678.

74. Cancer Genome Atlas Network. Comprehensive molecular portraits of human breast tumours. Nature. 2012;490(7418):61-70.

75. Nagy Á, et al. Validation of miRNA prognostic power in hepatocellular carcinoma using expression data of independent datasets. Sci Rep. 2018;8(1):9227. 